\title{
Induced QCD I: theory
}

\author{
Bastian B. Brandt, ${ }^{a, b}$ Robert Lohmayer $^{b}$ and Tilo Wettig ${ }^{b}$ \\ ${ }^{a}$ Institute for Theoretical Physics, Goethe-University of Frankfurt, \\ 60438 Frankfurt, Germany \\ ${ }^{b}$ Institute for Theoretical Physics, University of Regensburg, \\ 93040 Regensburg, Germany \\ E-mail: bastian.brandt@ur.de, robert.lohmayer@ur.de, tilo.wettig@ur.de
}

ABSTRACT: We explore an alternative discretization of continuum $\mathrm{SU}\left(N_{c}\right)$ Yang-Mills theory on a Euclidean spacetime lattice, originally introduced by Budzcies and Zirnbauer. In this discretization the self-interactions of the gauge field are induced by a path integral over $N_{b}$ auxiliary boson fields, which are coupled linearly to the gauge field. The main progress compared to earlier approaches is that $N_{b}$ can be as small as $N_{c}$. In the present paper we (i) extend the proof that the continuum limit of the new discretization reproduces Yang-Mills theory in two dimensions from gauge group $\mathrm{U}\left(N_{c}\right)$ to $\mathrm{SU}\left(N_{c}\right)$, (ii) derive refined bounds on $N_{b}$ for non-integer values, and (iii) perform a perturbative calculation to match the bare parameter of the induced gauge theory to the standard lattice coupling. In follow-up papers we will present numerical evidence in support of the conjecture that the induced gauge theory reproduces Yang-Mills theory also in three and four dimensions, and explore the possibility to integrate out the gauge fields to arrive at a dual formulation of lattice QCD.

KEYwords: Lattice QCD, Lattice Quantum Field Theory

ARXIV EPRINT: 1609.06466 


\section{Contents}

1 Introduction 2

2 Boson-induced pure gauge theory $\quad 2$

2.1 Formulation of Budczies and Zirnbauer 3

2.2 Alternative formulation without sign problem 4

3 Continuum limit for $\mathrm{U}\left(N_{c}\right)$ and $\mathrm{SU}\left(N_{c}\right) \quad 6$

$\begin{array}{ll}3.1 & \text { Character expansion and exponential parameterization }\end{array}$

$3.2 \delta$-function property 8

$\begin{array}{lll}3.2 .1 & G=\mathrm{U}(1) & 8\end{array}$

$\begin{array}{lll}3.2 .2 & G=\mathrm{SU}(2) & 9\end{array}$

$\begin{array}{lll}3.2 .3 G=\mathrm{U}\left(N_{c}\right) & 10\end{array}$

$\begin{array}{lll}3.2 .4 G=\mathrm{SU}\left(N_{c}\right) & 12\end{array}$

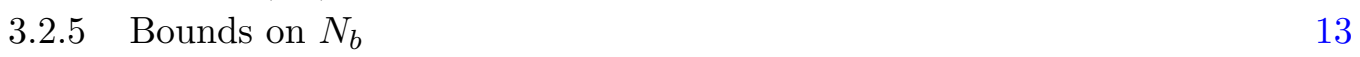

$\begin{array}{ll}3.3 & \text { Nature of the continuum limit } \\ \end{array}$

$\begin{array}{lll}\text { 3.3.1 NLO terms for } G=\mathrm{U}\left(N_{c}\right) & 14\end{array}$

$\begin{array}{lll}\text { 3.3.2 } & \text { NLO terms for } G=\mathrm{SU}\left(N_{c}\right) & 14\end{array}$

$\begin{array}{lll}\text { 3.3.3 Character expansion for } \mathrm{SU}\left(N_{c}\right) \text { Wilson plaquette action } & 15\end{array}$

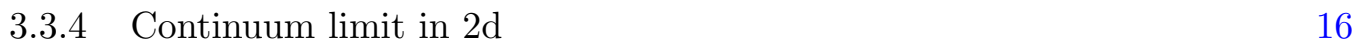

$\begin{array}{lll}3.3 .5 & \text { Bounds on } N_{b} \text { in } 2 \mathrm{~d} & 17\end{array}$

$\begin{array}{ll}3.3 .6 & \text { Continuum limit in } 3 \mathrm{~d} \text { and } 4 \mathrm{~d} \\ \end{array}$

4 Perturbative matching of the couplings $\quad 17$

$\begin{array}{lll}4.1 \text { General strategy } & 17\end{array}$

$\begin{array}{lll}4.2 & \text { Background-field calculation } & 20\end{array}$

$\begin{array}{lll}4.2 .1 & \text { Effective action } & 20\end{array}$

$\begin{array}{ll}\text { 4.2.2 Expansion of the gauge action } & 21\end{array}$

4.2.3 Gauge fixing and free action for the quantum field 22

$\begin{array}{lll}4.2 .4 & \text { Power counting } & 23\end{array}$

4.2.5 Effective action to one loop 24

\begin{tabular}{ll}
4.2 .6 & Relevant two-loop contributions \\
\hline
\end{tabular}

$\begin{array}{lll}4.3 & \text { Comparison with numerical results } & 29\end{array}$

$\begin{array}{llr}5 & \text { Conclusions } & 29\end{array}$

A Color traces $\quad 30$

B Character expansion for $\mathrm{SU}(2)$ and $\mathrm{U}(1) \quad 31$

$\begin{array}{lll}\text { B.1 } & \mathrm{SU}(2) & 31\end{array}$

B.2 $\mathrm{U}(1) \quad 33$

C Explicit expansion of the induced gauge action 33 


\section{Introduction}

Strong-coupling approaches to lattice gauge theories, in particular to lattice QCD, have a long history since they allow both for analytical investigations and for the construction of new simulation algorithms, see, e.g., [1-5]. Typically, these approaches work only if the selfinteraction of the gauge fields is neglected, giving rise to an uncontrolled systematic error of the results. There have been several ideas [6-9] to overcome this limitation by minimally coupling auxiliary degrees of freedom to the gauge field such that, after the auxiliary fields are integrated out, the correct gauge action is "induced" in a well-defined limit. However, in most cases this limit involves an infinite number of auxiliary fields, which is not useful for numerical simulations. An exception is the approach of Kazakov and Migdal (KM) [8], but the KM model does not induce the desired Yang-Mills (YM) theory since (i) the action has a local center symmetry, which forces all Wilson loops to vanish [10, 11], and (ii) an explicit solution with a quadratic potential showed that in this case the KM model does not yield the correct continuum behavior [12], see [13] for a review.

A major step forward was taken more than 10 years ago by Budczies and Zirnbauer (BZ) [14], who presented a novel method to induce the gauge action. The essential ideas of this method, which uses a small number $N_{b}$ of auxiliary bosons, will be reviewed in section 2.1. In short, the BZ method uses a "designer action" that couples the auxiliary bosons to the gauge field in such a way that, if the boson mass is taken to a critical value, the theory has the same continuum limit as YM theory provided that $N_{b}$ is larger than a certain lower bound. This was shown analytically for $d=2$ and gauge group $\mathrm{U}\left(N_{c}\right)$ by matching to an earlier result of Witten [15], while for $d>2$ there is no analytical proof but a plausible universality argument.

In this paper we study various theoretical aspects of the BZ method. In section 2 we reformulate the $\mathrm{BZ}$ action to eliminate a spurious sign problem. In section 3 we generalize it to the case of gauge group $\mathrm{SU}\left(N_{c}\right)$ and derive, for both $\mathrm{U}\left(N_{c}\right)$ and $\mathrm{SU}\left(N_{c}\right)$, precise bounds on $N_{b}$ for which a continuum limit exists and for which this limit corresponds to YM theory. In section 4 we perform a perturbative calculation for $\mathrm{SU}\left(N_{c}\right)$ to match the coupling in the BZ action to that of the standard Wilson plaquette action.

In follow-up papers we will present numerical evidence for the conjecture that the BZ method induces YM theory in the continuum limit by means of lattice simulations for $\mathrm{SU}(2)$ in $3 \mathrm{~d}$ and for $\mathrm{SU}(3)$ in $4 \mathrm{~d}$, and explore the construction of dual formulations of lattice QCD by applying the BZ method and integrating out the gauge and fermion fields. First reports of our study appeared in $[16,17]$.

\section{Boson-induced pure gauge theory}

In this section we review the basic idea of the BZ method and reformulate the action to eliminate a spurious sign problem. Unless stated otherwise, all dimensionful quantities are made dimensionless by multiplication with an appropriate power of the lattice spacing $a$, which we set to unity. 


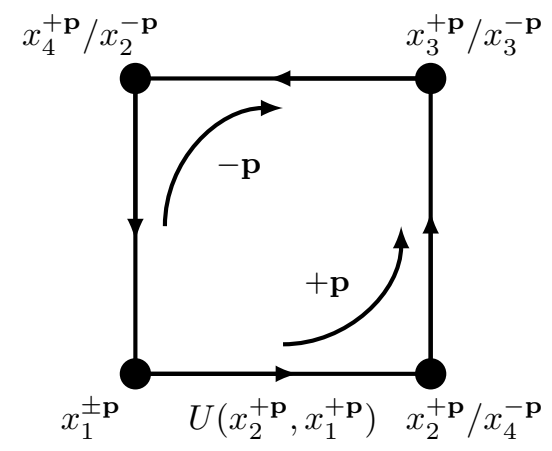

Figure 1. Conventions for the orientations of a plaquette and the corresponding lattice sites and link variables. With $U_{\mu}(x)=U(x+\mu, x)$ we have $U_{\mathbf{p}}=U\left(x_{1}^{\mathbf{p}}, x_{4}^{\mathbf{p}}\right) U\left(x_{4}^{\mathbf{p}}, x_{3}^{\mathbf{p}}\right) U\left(x_{3}^{\mathbf{p}}, x_{2}^{\mathbf{p}}\right) U\left(x_{2}^{\mathbf{p}}, x_{1}^{\mathbf{p}}\right)$ and a similar expression for $U_{-\mathbf{p}}$ with $\mathbf{p}$ replaced by $-\mathbf{p}$. Because of $U(x+\mu, x)=U(x, x+\mu)^{\dagger}$ we have $U_{-\mathbf{p}}=U_{\mathbf{p}}^{\dagger}$.

\subsection{Formulation of Budczies and Zirnbauer}

We restrict ourselves to gauge group $G=\mathrm{U}\left(N_{c}\right)$ or $\mathrm{SU}\left(N_{c}\right)$ in this paper. In [14] the gluonic weight for a configuration $[U]$ of gauge fields $U_{\mu}(x)$ in the fundamental representation of $G$ is taken to be

$$
\omega_{\mathrm{BZ}}[U] \sim \prod_{ \pm \mathbf{p}} \operatorname{det}\left(m_{\mathrm{BZ}}^{4}-U_{\mathbf{p}}\right)^{-N_{b}}=\prod_{p}\left|\operatorname{det}\left(m_{\mathrm{BZ}}^{4}-U_{p}\right)\right|^{-2 N_{b}}
$$

where here and below the symbol $\sim$ means that we have ignored a normalization factor that will be reinstated when it becomes important. The first product is over all oriented plaquettes (see figure 1 for our conventions), ${ }^{1}$ while the second product is over all unoriented plaquettes, where we have used $U_{-\mathbf{p}}=U_{\mathbf{p}}^{\dagger}$ and defined $U_{p}=U_{\mathbf{p}}$. (The definition $U_{p}=U_{-\mathbf{p}}$ would lead to the same final results.) As a special case of the more general discussion in [14], we take $m_{\mathrm{BZ}}$ to be real and identical for all plaquettes. Equation (2.1) implies that we need $m_{\mathrm{BZ}}>1$ to have a convergent theory.

Note that the weight factor (2.1) has the same global center-symmetry property as in YM theory due to the fact that the gauge fields only appear as full plaquettes in a class function in the action. This is of particular relevance for the deconfinement transition. The invariance under a local center symmetry is a major shortcoming of the KM model.

The expectation value of an observable $O[U]$ is given by the path integral

$$
\langle O\rangle=\frac{1}{Z} \int_{G}[d U] O[U] \omega_{\mathrm{BZ}}[U]
$$

with the partition function $Z$ defined by $\langle 1\rangle=1$. We follow the convention of [14] to use square brackets for a full set (i.e., a configuration) of gauge (or auxiliary) fields.

\footnotetext{
${ }^{1}$ For the sake of brevity and clarity we restrict ourselves to a hypercubic lattice. The discussion can straightforwardly be generalized to other lattice geometries using the framework and notation of [14].
} 
There is a "naive" pure-gauge limit in which the effective action resulting from (2.1) reduces to the Wilson plaquette action [18]. Writing $\omega_{\mathrm{BZ}}[U] \sim \exp \left(-S_{\mathrm{BZ}}^{\mathrm{eff}}[U]\right)$ we have [14]

$$
S_{\mathrm{BZ}}^{\mathrm{eff}}[U] \rightarrow S_{W}[U]=-\frac{\beta}{N_{c}} \sum_{p} \operatorname{Re} \operatorname{tr} U_{p}
$$

in the combined limit $m_{\mathrm{BZ}} \rightarrow \infty$ and $N_{b} \rightarrow \infty$ with $\beta=2 N_{c} N_{b} / m_{\mathrm{BZ}}^{4}$ fixed. The continuum limit then corresponds to taking the lattice coupling parameter $\beta$ to infinity. The naive limit requires large $N_{b}$, similar as in the earlier approaches $[6,7,9]$. However, the main point of the BZ method is that YM theory can also be obtained in the "interesting" limit $m_{\mathrm{BZ}} \rightarrow 1$ with $N_{b}$ fixed at a finite (and small) value. This will be the subject of section 3 .

To bosonize the determinants in (2.1) we assume that $N_{b}$ is a positive integer and introduce auxiliary boson fields $\varphi_{b, \mathbf{p}}\left(x_{j}^{\mathbf{p}}\right)$, where the index $b=1, \ldots, N_{b}$ labels the boson flavor, $x_{j}^{\mathbf{p}}$ with $j=1, \ldots, 4$ is defined in figure 1 , and the index $\mathbf{p}$ on $\varphi$ means that we have different fields for different (oriented) plaquettes. The fields are in the fundamental representation of $G$ and thus carry a color index that we did not write explicitly. Using these fields we have

$$
\omega_{\mathrm{BZ}}[U] \sim \int[d \varphi] \exp \left(-S_{\mathrm{BZ}}[\varphi, U]\right)
$$

with

$$
S_{\mathrm{BZ}}[\varphi, U]=\sum_{b=1}^{N_{b}} \sum_{ \pm \mathbf{p}} \sum_{j=1}^{4}\left[m_{\mathrm{BZ}} \varphi_{b, \mathbf{p}}^{\dagger}\left(x_{j}^{\mathbf{p}}\right) \varphi_{b, \mathbf{p}}\left(x_{j}^{\mathbf{p}}\right)-\varphi_{b, \mathbf{p}}^{\dagger}\left(x_{j+1}^{\mathbf{p}}\right) U\left(x_{j+1}^{\mathbf{p}}, x_{j}^{\mathbf{p}}\right) \varphi_{b, \mathbf{p}}\left(x_{j}^{\mathbf{p}}\right)\right],
$$

where $x_{5}^{\mathbf{p}} \equiv x_{1}^{\mathbf{p}}$. The connection between (2.1) and (2.4) is readily shown by rewriting the action (2.5) in bilinear form, integrating out the boson fields, and converting the matrix in the resulting determinant to upper-triangular form. From (2.5) it is clear that the parameter $m_{\mathrm{BZ}}$ is the mass of the auxiliary bosons and that the total number of boson fields per plaquette is $2 N_{b}$.

\subsection{Alternative formulation without sign problem}

One of the interesting aspects of the BZ method is that it can lead to reformulations of lattice QCD. To make numerical simulations feasible it is important to have a real action so that the weight factor is real and positive. While the weight factor (2.1), in which the bosons are integrated out, satisfies this criterion, this is no longer true for the action (2.5). In this case the action is generically complex since the imaginary parts of the terms containing the positively and negatively oriented links only cancel after averaging over the boson fields.

The sign problem in the action (2.5) would not be present if the two hopping terms including a particular link $U_{\mu}(x)$, i.e., the terms

$$
\varphi_{b,+\mathbf{p}}^{\dagger}(x+\mu) U_{\mu}(x) \varphi_{b,+\mathbf{p}}(x) \quad \text { and } \quad \varphi_{b,-\mathbf{p}}^{\dagger}(x) U_{\mu}(x)^{\dagger} \varphi_{b,-\mathbf{p}}(x+\mu)
$$


were complex conjugates of each other so that their sum is real. This can be achieved by assigning the boson fields to unoriented (rather than oriented) plaquettes and using the alternative action

$$
\begin{aligned}
S_{B}[\varphi, U]=\sum_{b=1}^{N_{b}} \sum_{p} \sum_{j=1}^{4}[ & m \varphi_{b, p}^{\dagger}\left(x_{j}^{p}\right) \varphi_{b, p}\left(x_{j}^{p}\right)-\varphi_{b, p}^{\dagger}\left(x_{j+1}^{p}\right) U\left(x_{j+1}^{p}, x_{j}^{p}\right) \varphi_{b, p}\left(x_{j}^{p}\right) \\
& \left.-\varphi_{b, p}^{\dagger}\left(x_{j}^{p}\right) U\left(x_{j}^{p}, x_{j+1}^{p}\right) \varphi_{b, p}\left(x_{j+1}^{p}\right)\right]
\end{aligned}
$$

with $x_{j}^{p}=x_{j}^{+\mathbf{p}}$. Note that we now have only half the number of boson fields compared to the action (2.5). The matrix associated with the bilinear form in the boson fields is Hermitian, leading to a real action and thus a positive definite weight

$$
\omega[\varphi, U] \sim \exp \left(-S_{B}[\varphi, U]\right)
$$

for all gauge and boson field configurations.

Some algebra is needed to show that the two actions (2.5) and (2.7) are equivalent. The first step is to integrate out the boson fields in the path integral associated with the action (2.7). This yields the inverse determinant of the matrix corresponding to the bilinear form in the boson fields. This matrix is diagonal in $b$ and $p$ so that its determinant factorizes into a product of determinants of $4 N_{c} \times 4 N_{c}$ matrices, with the product running over plaquettes and boson flavors. These determinants can be evaluated by converting the $4 \times 4$ part with $N_{c} \times N_{c}$ matrices as entries to upper triangular form. The final result is

$$
\omega[U] \sim \prod_{p} \operatorname{det}\left(1-\frac{\alpha}{2}\left(U_{p}+U_{p}^{\dagger}\right)\right)^{-N_{b}}
$$

with $^{2}$

$$
\frac{2}{\alpha}=m^{4}-4 m^{2}+2
$$

The weight factors (2.1) and (2.9) are directly related via

$$
\begin{aligned}
\left|\operatorname{det}\left(m_{\mathrm{BZ}}^{4}-U_{p}\right)\right|^{2} & =\operatorname{det}\left(\left(m_{\mathrm{BZ}}^{4}-U_{p}\right)\left(m_{\mathrm{BZ}}^{4}-U_{p}^{\dagger}\right)\right) \\
& \sim \operatorname{det}\left(\frac{2}{\alpha}-\left(U_{p}+U_{p}^{\dagger}\right)\right)
\end{aligned}
$$

if we identify

$$
\frac{2}{\alpha}=m_{\mathrm{BZ}}^{4}+m_{\mathrm{BZ}}^{-4}
$$

From (2.12) and $m_{\mathrm{BZ}}>1$ we obtain the condition $0<\alpha<1$. Via (2.10) this implies $m>2$ in (2.7). The limit $m_{\mathrm{BZ}} \rightarrow 1^{+}$corresponds to the limit $\alpha \rightarrow 1^{-}$(or, equivalently, $\left.m \rightarrow 2^{+}\right)$. From now on we will use the weight factor (2.9).

\footnotetext{
${ }^{2}$ Note that the result (2.10) for $\alpha$ is only valid for hypercubic lattices. The relation between $\alpha$ and $m$ will be different for other lattice structures.
} 


\section{Continuum limit for $\mathrm{U}\left(N_{c}\right)$ and $\mathrm{SU}\left(N_{c}\right)$}

In [14] it was shown, for $G=\mathrm{U}\left(N_{c}\right)$, that a continuum limit exists for $\alpha \rightarrow 1$ provided that $N_{b} \geq N_{c}$, and that in two dimensions this continuum limit is in the YM universality class if $N_{b} \geq N_{c}+1$. For three or more dimensions it was conjectured, based on plausible universality arguments, that the continuum limit is in the YM universality class if $N_{b} \geq N_{c}$.

In this section we do not assume that $N_{b}$ is integer and also consider $G=\mathrm{SU}\left(N_{c}\right)$. In section 3.1 we set up the basic mathematical framework. In section 3.2 we derive refined bounds on $N_{b}$ for which the gauge theory with weight function (2.9) has a continuum limit for $\alpha \rightarrow 1$. In section 3.3 we derive bounds on $N_{b}$ which ensure that the continuum limit is in the YM universality class. The main results are given in (3.38) and (3.54).

\subsection{Character expansion and exponential parameterization}

Let us write the gluonic weight (2.9) in the form

$$
\omega[U] \sim \prod_{p} \omega\left(U_{p}\right) \quad \text { with } \quad \omega(U)=\frac{1}{Z(\alpha)} \operatorname{det}\left(1-\frac{\alpha}{2}\left(U+U^{\dagger}\right)\right)^{-N_{b}} .
$$

For simplicity of notation we denote the weights for the ensemble of gauge fields and for a single plaquette by the same symbol $\omega$. The distinction between the two cases is made by the square or round brackets. The factor $Z(\alpha)$ ensures that $\omega(U)$ is properly normalized and is therefore given by

$$
Z(\alpha)=\int_{G} d U \operatorname{det}\left(1-\frac{\alpha}{2}\left(U+U^{\dagger}\right)\right)^{-N_{b}}
$$

where $d U$ denotes the Haar measure, normalized such that $\int_{G} d U=1$.

Since $\omega(U)$ is a class function we can expand it in the characters of the irreducible representations (or irreps) of $G$,

$$
\omega(U)=\sum_{\lambda} c_{\lambda}(\alpha) \chi_{\lambda}(U)
$$

where $\lambda$ labels the inequivalent irreps and $\chi_{\lambda}(U)$ is the character of $U$ in $\lambda$. Using character orthogonality,

$$
\int_{G} d U \chi_{\lambda}(U V) \chi_{\gamma}\left(U^{\dagger} W\right)=\delta_{\lambda \gamma} \frac{1}{d_{\lambda}} \chi_{\lambda}(V W)
$$

with $d_{\lambda}=\operatorname{dim}(\lambda)$, the coefficients are given by

$$
c_{\lambda}(\alpha)=\int_{G} d U \omega(U) \chi_{\lambda}\left(U^{\dagger}\right)
$$

Clearly $c_{0}(\alpha)=1$, where $\lambda=0$ labels the trivial representation. When $\lambda$ is not specified, $\operatorname{det}(\cdot)$ and $\operatorname{tr}(\cdot)$ always refer to the fundamental representation.

To compute the integral (3.5) we use an exponential parameterization of the form

$$
U=\exp (i \sqrt{\gamma} H) \quad \text { with } \gamma=\frac{2}{\alpha}(1-\alpha) \text { and } H \in \mathfrak{g},
$$


where $\mathfrak{g}$ denotes the group algebra of $G$. The factor of $\sqrt{\gamma}$ was chosen such that the parameterization (3.6) leads to a systematic expansion of the $U$-dependent part of the weight function in powers of $(1-\alpha)$ for fixed $H$,

$$
\begin{aligned}
\operatorname{det}\left(1-\frac{\alpha}{2}\left(U+U^{\dagger}\right)\right) & =\operatorname{det}(1-\alpha \cos (\sqrt{\gamma} H)) \\
& =(1-\alpha)^{N_{c}} \operatorname{det}\left(1+H^{2}-\frac{\gamma}{12} H^{4}+\ldots\right),
\end{aligned}
$$

where the higher-order terms in the determinant are of the form $\gamma^{k} H^{2 k+2}$ with $k \geq 2$.

The integration measure becomes (see, e.g., [19, appendix C])

$$
d U=\gamma^{n / 2} \sqrt{\operatorname{det} g(H)} d H \quad \text { with } \quad g(H)=\sum_{\ell=0}^{\infty} \frac{(-1)^{\ell} \gamma^{\ell}}{(2 \ell+2) !} \mathcal{H}^{2 \ell},
$$

where

$$
n= \begin{cases}N_{c}^{2} & \text { for } G=\mathrm{U}\left(N_{c}\right), \\ N_{c}^{2}-1 & \text { for } G=\mathrm{SU}\left(N_{c}\right)\end{cases}
$$

is the number of generators of $G, \mathcal{H}=\sum_{a=1}^{n} h_{a} t_{a}^{(\operatorname{adj})}$ denotes the element of the adjoint representation of $\mathfrak{g}$ corresponding to $H=\sum_{a=1}^{n} h_{a} t_{a}^{\text {(fund) }}$ in the fundamental representation, and the $t_{a}$ are the generators of the representation normalized according to (A.1). The integral over $H$ is defined as an $n$-dimensional integral over the coefficients $h_{a}$, i.e.,

$$
d H=\prod_{a=1}^{n} d h_{a}
$$

The integration domain $V(\gamma, \mathfrak{g})$ is chosen such that the group $G$ is covered exactly once (or a finite number of times, resulting in an irrelevant normalization factor). Note that this domain $V(\gamma, \mathfrak{g})$ depends on $\gamma$. In fact, we will only have to evaluate integrals of class functions, where the appropriate integration domains for the eigenvalues of $U$ are obvious.

For the expansion of the character we have [14, 20]

$$
\frac{\chi_{\lambda}\left(e^{-i \sqrt{\gamma} H}\right)}{d_{\lambda}}=1-i \sqrt{\gamma} \frac{q(\lambda)}{N_{c}} \operatorname{tr} H-\frac{\gamma}{2}\left(\left(\frac{q(\lambda)^{2}}{N_{c}^{2}}-\frac{A(\lambda)}{N_{c}}\right)(\operatorname{tr} H)^{2}+A(\lambda) \operatorname{tr} H^{2}\right)+\ldots
$$

with $^{3}$

$$
\begin{aligned}
A(\lambda) & =\frac{2}{N_{c}^{2}-1}\left(C_{2}^{\mathrm{U}}(\lambda)-\frac{q(\lambda)^{2}}{2 N_{c}}\right), \\
q(\lambda) & =\sum_{j=1}^{N_{c}} \lambda_{j}, \\
C_{2}^{\mathrm{U}}(\lambda) & =\frac{1}{2} \sum_{j=1}^{N_{c}} \lambda_{j}\left(\lambda_{j}+N_{c}+1-2 j\right) .
\end{aligned}
$$

\footnotetext{
${ }^{3}$ Note that our $C_{2}^{\mathrm{U}}(\lambda)$ differs from [14] by a factor of 2 since we use the standard normalization (A.1).
} 
Here, the ordered set of integers $\lambda_{1} \geq \lambda_{2} \geq \ldots \geq \lambda_{N_{c}}$ defines the irrep $\lambda$ of $G[20],{ }^{4}$ and $C_{2}^{\mathrm{U}}(\lambda)$ is the quadratic Casimir invariant of $\mathrm{U}\left(N_{c}\right)$. The second factor on the r.h.s. of (3.12) is the quadratic Casimir invariant of $\mathrm{SU}\left(N_{c}\right)$ [21],

$$
C_{2}^{\mathrm{SU}}(\lambda)=C_{2}^{\mathrm{U}}(\lambda)-\frac{q(\lambda)^{2}}{2 N_{c}}
$$

For $G=\mathrm{SU}\left(N_{c}\right)$ we have $\operatorname{tr} H=0$ so that (3.11) simplifies to

$$
\frac{\chi_{\lambda}\left(e^{-i \sqrt{\gamma} H}\right)}{d_{\lambda}}=1-\frac{C_{2}^{\mathrm{SU}}(\lambda)}{N_{c}^{2}-1} \gamma \operatorname{tr} H^{2}+\ldots, \quad G=\mathrm{SU}\left(N_{c}\right) .
$$

We now pull out a trivial factor of $(\gamma / 2)^{n / 2}(1-\alpha)^{-N_{b} N_{c}}$ from both $Z(\alpha)$ in (3.2) and $d U \operatorname{det}(1-\alpha \cos (\sqrt{\gamma} H))^{-N_{b}}$ in (3.5) and obtain

$$
\begin{aligned}
c_{\lambda}(\alpha) & =\frac{\bar{c}_{\lambda}(\gamma)}{\bar{c}_{0}(\gamma)}, \\
\bar{c}_{\lambda}(\gamma) & =\int_{V(\gamma, \mathfrak{g})} d H \bar{\omega}(H) \chi_{\lambda}\left(e^{-i \sqrt{\gamma} H}\right), \\
\bar{\omega}(H) & =\sqrt{\operatorname{det}(2 g(H))} \operatorname{det}\left(\frac{1-\alpha \cos (\sqrt{\gamma} H)}{1-\alpha}\right)^{-N_{b}},
\end{aligned}
$$

where $\alpha$ and $\gamma$ are related by (3.6). Note that

$$
\begin{aligned}
\lim _{\alpha \rightarrow 1} \sqrt{\operatorname{det}(2 g(H))} & =1, \\
\lim _{\alpha \rightarrow 1} \bar{\omega}(H) & =\operatorname{det}\left(1+H^{2}\right)^{-N_{b}}, \\
\lim _{\alpha \rightarrow 1} \chi_{\lambda}\left(e^{-i \sqrt{\gamma} H}\right) & =\chi_{\lambda}(\mathbb{1})=d_{\lambda} .
\end{aligned}
$$

\section{$3.2 \delta$-function property}

As explained in [14], a continuum limit is obtained if $\omega(U)$ approaches a $\delta$-function located at $U=\mathbb{1}$. As a consequence of the Peter-Weyl theorem, the character expansion of the $\delta$-function is given by (3.3) with $c_{\lambda}(\alpha)$ replaced by the dimension $d_{\lambda}$ of the irrep $\lambda$. Hence, $\omega(U)$ becomes a $\delta$-function if $\lim _{\alpha \rightarrow 1} c_{\lambda}(\alpha)=d_{\lambda}$ for all $\lambda$. We now investigate under what conditions this criterion is satisfied for the different gauge groups.

\subsection{1 $G=\mathrm{U}(1)$}

It is instructive to first study the simplest case $G=\mathrm{U}(1)$ in some detail. In this case, (3.18) reduces to the one-dimensional integral

$$
\bar{c}_{\lambda}(\gamma)=\int_{0}^{\pi / \sqrt{\gamma}} d x \frac{1}{\left(1+x^{2}\right)^{N_{b}}} \sum_{k, m=0}^{\infty} a_{k, m}(\lambda) \gamma^{k+m}\left(\frac{x^{4}}{1+x^{2}}\right)^{k} x^{2 m}
$$

\footnotetext{
${ }^{4}$ Two irreps $\lambda$ and $\mu$ related by $\lambda_{j}=\mu_{j}+m$ with $m \in \mathbb{Z}$ only differ by a factor of $\operatorname{det}(U)^{m}$. For $G=\mathrm{SU}\left(N_{c}\right)$ we have $\operatorname{det} U=1$. In this case $\lambda$ and $\mu$ are equivalent, and the inequivalent irreps are conventionally chosen to be those with $\lambda_{N_{c}}=0$. Then all inequivalent irreps $\lambda$ are given by Young diagrams with $N_{c}-1$ rows, with $\lambda_{j} \geq 0$ equal to the length of row $j$.
} 
with some coefficients $a_{k, m}(\lambda)$. The asymptotic behavior of $\bar{c}_{\lambda}(\gamma)$ as $\alpha \rightarrow 1$, i.e., $\gamma \rightarrow 0$, is therefore determined by integrals of the form

$$
I_{k, m}(\gamma)=\gamma^{k+m} \int_{0}^{\pi / \sqrt{\gamma}} d x \frac{x^{2(k+m)+2 k}}{\left(1+x^{2}\right)^{N_{b}+k}}
$$

The integral in (3.22) is finite as $\gamma \rightarrow 0$ as long as $N_{b}>k+m+\frac{1}{2}$. The limit is

$$
\int_{0}^{\infty} d x \frac{x^{4 k+2 m}}{\left(1+x^{2}\right)^{N_{b}+k}}=\frac{\Gamma\left(2 k+m+\frac{1}{2}\right) \Gamma\left(N_{b}-k-m-\frac{1}{2}\right)}{2 \Gamma\left(N_{b}+k\right)} .
$$

For $N_{b}<k+m+\frac{1}{2}$, the integral in (3.22) diverges like $\gamma^{-\left(k+m+\frac{1}{2}-N_{b}\right)}$. If $N_{b}=k+m+\frac{1}{2}$, we obtain a logarithmic divergence. Hence, the leading-order behavior of $I_{k, m}(\gamma)$ as $\gamma \rightarrow 0$ is given by

$$
I_{k, m}(\gamma) \propto \begin{cases}\gamma^{k+m} & \text { for } k+m<N_{b}-\frac{1}{2}, \\ \gamma^{N_{b}-\frac{1}{2}} \log \gamma & \text { for } k+m=N_{b}-\frac{1}{2}, \\ \gamma^{N_{b}-\frac{1}{2}} & \text { for } k+m>N_{b}-\frac{1}{2} .\end{cases}
$$

From (3.24) we see that for $N_{b}>\frac{1}{2}, \bar{c}_{\lambda}(\gamma)$ is dominated by the finite and non-zero term with $k=m=0$ in (3.21), with corrections of order $\mathcal{O}\left(\gamma^{\min \left(1, N_{b}-\frac{1}{2}\right)}\right)$, or $\mathcal{O}(\gamma \log \gamma)$ for $N_{b}=\frac{3}{2}$. For $N_{b}=\frac{1}{2}$, the term with $k=m=0$ diverges like $\log \gamma$ while all other terms are finite as $\gamma \rightarrow 0$. Therefore, for $N_{b} \geq \frac{1}{2}$ we have

$$
\lim _{\alpha \rightarrow 1} c_{\lambda}(\alpha)=\lim _{\gamma \rightarrow 0} \frac{\bar{c}_{\lambda}(\gamma)}{\bar{c}_{0}(\gamma)}=\frac{a_{0,0}(\lambda)}{a_{0,0}(0)}=d_{\lambda}=1,
$$

where the penultimate equality follows from (3.20). This implies that for $N_{b} \geq \frac{1}{2}$ the weight function $\omega(U)$ reduces to a $\delta$-function on the $\mathrm{U}(1)$ manifold in the limit $\alpha \rightarrow 1$.

On the other hand, for $N_{b}<\frac{1}{2}$ we have $k+m \geq 0>N_{b}-\frac{1}{2}$ for all terms, i.e., for all $k$ and $m$ we obtain the same leading divergence, $I_{k, m} \propto \gamma^{-\left(\frac{1}{2}-N_{b}\right)}$. Therefore, all terms in the sum over $k$ and $m$ contribute to $\lim _{\alpha \rightarrow 1} c_{\lambda}(\alpha)$, making the dependence on $\lambda$ non-trivial, and consequently $\lim _{\alpha \rightarrow 1} c_{\lambda}(\alpha) \neq d_{\lambda}$ generically. Hence we obtain a $\delta$-function if and only if $N_{b} \geq \frac{1}{2}$.

For $N_{b} \in \mathbb{N}$, the coefficients $c_{\lambda}$ can be calculated analytically as a function of $\alpha$, see appendix B.2. The results are consistent with the condition $N_{b} \geq \frac{1}{2}$.

\subsection{2 $G=\mathrm{SU}(2)$}

Parametrizing $H \in \mathfrak{s u}(2)$ in terms of its eigenvalues, $H=V \operatorname{diag}(x,-x) V^{\dagger}$ with $V \in$ $\mathrm{SU}(2)$, leads to $\operatorname{det}\left(1+H^{2}\right)=\left(1+x^{2}\right)^{2}$ and integration measure $d H \propto(x-(-x))^{2}$ (see section 3.2.4 below). The coefficient $\bar{c}_{\lambda}$ is then given by a single integral equivalent to the integral (3.21) except for the replacement $\left(1+x^{2}\right)^{-N_{b}} \rightarrow x^{2}\left(1+x^{2}\right)^{-2 N_{b}}$. We can therefore immediately apply the power-counting arguments of the previous section after substituting $N_{b} \rightarrow 2 N_{b}-1$. This results in $N_{b} \geq \frac{3}{4}$ as a necessary and sufficient condition for $\omega(U)$ to approach the $\delta$-function as $\alpha \rightarrow 1$.

For $2 N_{b} \in \mathbb{N}$, the coefficients $c_{\lambda}$ can be calculated analytically as a function of $\alpha$, see appendix B.1. The results are consistent with the condition $N_{b} \geq \frac{3}{4}$. 


\subsection{3 $G=\mathrm{U}\left(N_{c}\right)$}

Since $H$ is Hermitian we transform to the eigenvalue representation $H=V \operatorname{diag}\left(x_{j}\right) V^{\dagger}$ with $x_{j}\left(1 \leq j \leq N_{c}\right)$ real and $V$ unitary. The Jacobian $J$ of this transformation is given by the square of a Vandermonde determinant, $J=\prod_{j<k}\left(x_{j}-x_{k}\right)^{2}$.

Let us first determine the asymptotic behavior (for $\gamma \rightarrow 0$ ) of the integral of the determinant (3.20b) over the domain $V\left(\gamma, \mathfrak{u}\left(N_{c}\right)\right)$,

$$
\int_{V\left(\gamma, \mathfrak{u}\left(N_{c}\right)\right)} d H \operatorname{det}\left(1+H^{2}\right)^{-N_{b}} \propto \int_{-\pi / \sqrt{\gamma}}^{\pi / \sqrt{\gamma}}\left(\prod_{j=1}^{N_{c}} d x_{j}\right)\left(\prod_{j<k}\left(x_{j}-x_{k}\right)^{2}\right)\left(\prod_{j=1}^{N_{c}}\left(1+x_{j}^{2}\right)^{-N_{b}}\right) .
$$

We now split the integral over the eigenvalues $x_{j}$ into several integrals over subdomains and separately analyze their asymptotic behavior by simple power counting:

(i) In domains where all $x_{j}$ are "finite" (in the sense that they are of order $\gamma^{0}$ and do not go to infinity like $1 / \sqrt{\gamma}$ ), the contributions to the integral are finite, i.e., of order $\gamma^{0}$.

(ii) Choose an integer $k$ with $0 \leq k \leq N_{c}-1$. In domains where $k$ of the variables (say, the $x_{i}$ with $\left.1 \leq i \leq k\right)$ stay "finite" and the remaining $N_{c}-k$ variables $\left(x_{i}\right.$, $k+1 \leq i \leq N_{c}$ ) are "large" (in the sense that they go to infinity like $1 / \sqrt{\gamma}$ ) and "independent" (in the sense that generically differences $x_{i}-x_{j}$ for $k+1 \leq i<j \leq N_{c}$ are "large"), the leading-order contributions to the integral are proportional to

$$
\left(\frac{1}{\sqrt{\gamma}}\right)^{N_{c}-k+2\left[\left(\begin{array}{c}
N_{c} \\
2
\end{array}\right)-\left(\begin{array}{c}
k \\
2
\end{array}\right)\right]-2 N_{b}\left(N_{c}-k\right)}=\gamma^{\frac{1}{2}\left(k-N_{b}\right)^{2}-\frac{1}{2}\left(N_{c}-N_{b}\right)^{2}}
$$

provided the exponent on the r.h.s. is negative (otherwise, the integral is finite). If $k=2 N_{b}-N_{c}$ is an integer satisfying $0 \leq k \leq N_{c}-1$, the integral diverges logarithmically.

(iii) If some of the $N_{c}-k$ large integration variables are not "independent", the possible degree of divergence is reduced compared to (3.27) since the effective number of large integration variables is reduced and some differences $x_{i}-x_{j}$ stay finite.

We therefore conclude

$$
\int_{V\left(\gamma, \mathfrak{u}\left(N_{c}\right)\right)} d H \operatorname{det}\left(1+H^{2}\right)^{-N_{b}} \propto \gamma^{\min \left\{\frac{1}{2}\left(k-N_{b}\right)^{2}-\frac{1}{2}\left(N_{c}-N_{b}\right)^{2}\right\}_{0 \leq k \leq N_{c}},}
$$

unless the minimum in the exponent on the r.h.s. equals zero and the corresponding $k$ satisfies $k \neq N_{c}$, in which case the integral diverges like $\log (\gamma)$. The minimal exponent in (3.28) is obtained for $k=\min \left\{\left[N_{b}\right], N_{c}\right\}$, where $\left[N_{b}\right]$ denotes the integer closest to $N_{b}$. Hence

$$
\int_{V\left(\gamma, \mathfrak{u}\left(N_{c}\right)\right)} d H \operatorname{det}\left(1+H^{2}\right)^{-N_{b}} \propto \begin{cases}\gamma^{\frac{1}{2}\left(\left[N_{b}\right]-N_{b}\right)^{2}-\frac{1}{2}\left(N_{c}-N_{b}\right)^{2}} & \text { for } N_{b}<N_{c}-\frac{1}{2} \\ \log (\gamma) & \text { for } N_{b}=N_{c}-\frac{1}{2} \\ \gamma^{0} & \text { for } N_{b}>N_{c}-\frac{1}{2}\end{cases}
$$


where the logarithmic divergence for $N_{b}=N_{c}-\frac{1}{2}$ results from $k=N_{c}-1$, i.e., an integration domain where one eigenvalue is "large" and all others remain "finite".

The result (3.29), which was obtained from simple power counting, could potentially be invalid. After we expand the Vandermonde determinant in (3.26), we obtain a sum of factorized integrals, all of the U(1) type (3.21). Cancellations in this sum could make the coefficient of the leading-order contribution to the integral vanish. We have explicitly checked for a range of values for $N_{c}$ that this does not happen. This confirms the validity of the simple power-counting arguments.

In complete analogy to the $\mathrm{U}(1)$ example, we expand the integrand in the integral representation (3.18) of $\bar{c}_{\lambda}(\gamma)$ in powers of $\gamma$. From (3.7), (3.8), and (3.11) we obtain

$$
\bar{c}_{\lambda}(\gamma)=d_{\lambda} \int_{V\left(\gamma, \mathfrak{u}\left(N_{c}\right)\right.} d H \operatorname{det}\left(1+H^{2}\right)^{-N_{b}}\left(1+\sum_{m=1}^{\infty} \gamma^{m} b_{\lambda, m}(H)\right)
$$

where the functions $b_{\lambda, m}(H)$ are of order $H^{2 m}$ and depend only on the eigenvalues $x_{i}$ of $H$. They do not depend on $\gamma$. A term contributing to $b_{\lambda, m}(H)$ is, e.g., given by $\left(\operatorname{tr} \frac{H^{4}}{1+H^{2}}\right)^{m}$, resulting from the expansion (3.7). To determine the asymptotic behavior of $\gamma^{m} \int_{V\left(\gamma, \mathfrak{u}\left(N_{c}\right)\right.} d H \operatorname{det}\left(1+H^{2}\right)^{-N_{b}} b_{\lambda, m}(H)$ at leading order as $\gamma \rightarrow 0$, we again analyze the different integration domains discussed above and include an additional factor of $\left(\gamma x_{N c}^{2}\right)^{m}$ in (3.26) $\left(x_{N_{c}}\right.$ always corresponds to a "large" variable in (ii) above and reflects the fact that the $b_{\lambda, m}(H)$ are of order $\left.H^{2 m}\right)$. For $m>0$ we obtain

$$
\begin{aligned}
\gamma^{m} \int_{V\left(\gamma, \mathfrak{u}\left(N_{c}\right)\right)} d H \operatorname{det}\left(1+H^{2}\right)^{-N_{b}} b_{\lambda, m}(H) & \\
\propto & \begin{cases}\gamma^{\frac{1}{2}\left(\left[N_{b}\right]-N_{b}\right)^{2}-\frac{1}{2}\left(N_{c}-N_{b}\right)^{2}} & \text { for } 0<N_{b}<N_{c}-\frac{1}{2}, \\
\gamma^{N_{b}-\left(N_{c}-\frac{1}{2}\right)} & \text { for } N_{c}-\frac{1}{2} \leq N_{b}<N_{c}+m-\frac{1}{2}, \\
\gamma^{m} \log (\gamma) & \text { for } N_{b}=N_{c}+m-\frac{1}{2}, \\
\gamma^{m} & \text { for } N_{c}+m-\frac{1}{2}<N_{b} .\end{cases}
\end{aligned}
$$

Note that for $m=0(3.31)$ reduces to (3.29) if we define $b_{\lambda, 0}(H)=1$. We can now analyze the dependence on $N_{b}$ of the series expansion of $\bar{c}_{\lambda}$ in powers of $\gamma$.

For $N_{b}>N_{c}-\frac{1}{2}$, the integral (3.31) is finite for all $m \geq 0$. While (3.31) with $m=0$ results in a contribution of order $\gamma^{0}$ in the expansion of $\bar{c}_{\lambda}$, all other $m \geq 1$ lead to contributions that vanish as $\gamma \rightarrow 0$ (terms of order $\gamma^{N_{b}-\left(N_{c}-\frac{1}{2}\right)}, \gamma^{m} \log \gamma$, or $\gamma^{m}$ ).

For $N_{b}=N_{c}-\frac{1}{2}$, the integral (3.31) diverges logarithmically for $m=0$, while $m \geq 1$ leads to finite terms $\left(\gamma^{N_{b}-\left(N_{c}-\frac{1}{2}\right)}=\gamma^{0}\right)$, i.e., the contribution of $m=0$ still dominates.

From (3.20) we then immediately obtain the straightforward generalization of (3.25),

$$
\lim _{\alpha \rightarrow 1} c_{\lambda}(\alpha)=\lim _{\gamma \rightarrow 0} \frac{\bar{c}_{\lambda}(\gamma)}{\bar{c}_{0}(\gamma)}=\frac{d_{\lambda}}{d_{0}}=d_{\lambda} \quad \text { for } N_{b} \geq N_{c}-\frac{1}{2} .
$$

For $N_{b}<N_{c}-\frac{1}{2}$, the integral (3.31) leads to identical leading-order divergences proportional to $\gamma^{\frac{1}{2}\left(\left[N_{b}\right]-N_{b}\right)^{2}-\frac{1}{2}\left(N_{c}-N_{b}\right)^{2}}$ for all $m$. In this case, $\lim _{\alpha \rightarrow 1} c_{\lambda}(\alpha)$ has a non-trivial dependence on $\lambda$ and therefore differs from $d_{\lambda}$ generically. 


\subsection{4 $G=\mathrm{SU}\left(N_{c}\right)$}

We again transform to the eigenvalue representation $H=V \operatorname{diag}\left(x_{j}\right) V^{\dagger}$. Since $H$ is now traceless we need to incorporate the condition $\sum_{j} x_{j}=0$ in the integral. This leads to an additional $\delta$-function,

$$
\begin{aligned}
& \int_{V\left(\gamma, \mathfrak{s u}\left(N_{c}\right)\right)} d H \operatorname{det}\left(1+H^{2}\right)^{-N_{b}} \\
& \propto \int_{-\pi / \sqrt{\gamma}}^{\pi / \sqrt{\gamma}}\left(\prod_{j=1}^{N_{c}} d x_{j}\right) \delta\left(\sum_{j=1}^{N_{c}} x_{j}\right)\left(\prod_{j<k}\left(x_{j}-x_{k}\right)^{2}\right)\left(\prod_{j=1}^{N_{c}}\left(1+x_{j}^{2}\right)^{-N_{b}}\right) \\
& \quad=\left.\int_{-\pi / \sqrt{\gamma}}^{\pi / \sqrt{\gamma}}\left(\prod_{j=1}^{N_{c}-1} d x_{j}\right)\left(\prod_{j<k}\left(x_{j}-x_{k}\right)^{2}\right)\left(\prod_{j=1}^{N_{c}}\left(1+x_{j}^{2}\right)^{-N_{b}}\right)\right|_{x_{N_{c}}=-\sum_{\ell=1}^{N_{c}-1} x_{\ell}} .
\end{aligned}
$$

In analogy with the previous subsection, we now evaluate the integral (3.33) with an additional factor of $x_{N_{c}-1}^{2 m}(m \geq 0)$ in the integrand to determine the equivalent of (3.31) for the $\mathrm{SU}\left(N_{c}\right)$ case. To this end, we again proceed by splitting the integral into integrals over subdomains and analyzing their asymptotic behavior by power counting:

(i) When all $x_{j}$ are "finite" the integral is finite, i.e., of order $\gamma^{0}$.

(ii) We choose an integer $k$ with $0 \leq k \leq N_{c}-2$ and consider domains where $k$ variables $\left(x_{i}, 1 \leq i \leq k\right)$ stay "finite" and $N_{c}-k-1$ variables $\left(x_{i}, k+1 \leq i \leq N_{c}-1\right)$ are "large". To obtain the largest possible degree of divergence, we have chosen the "large" variables such that the factor of $x_{N_{c}-1}^{2 m}$ always corresponds to a "large" variable. Furthermore, we take all large variables to be "independent" (in the same sense as before, except that we also require $x_{N_{c}}=-\sum_{i=1}^{N_{c}-1} x_{i}$ and all differences $x_{i}-x_{N_{c}}$ to be "large"). Then the leading-order contribution of such a domain to the integral is proportional to

$$
\left(\frac{1}{\sqrt{\gamma}}\right)^{N_{c}-k-1+2\left[\left(\begin{array}{c}
N_{c} \\
2
\end{array}\right)-\left(\begin{array}{c}
k \\
2
\end{array}\right)\right]-2 N_{b}\left(N_{c}-k\right)+2 m}=\gamma^{\frac{1}{2}\left(k-N_{b}\right)^{2}-\frac{1}{2}\left(N_{c}-N_{b}\right)^{2}+\frac{1}{2}-m},
$$

provided the exponent on the r.h.s. is negative (otherwise, the integral is finite). If the exponent vanishes, the integral diverges logarithmically.

(iii) If some of the $N_{c}-k-1$ "large" integration variables are not "independent" such that some differences $x_{i}-x_{j}$ stay finite, the possible degree of divergence is reduced compared to (3.34).

(iv) If some of the $N_{c}-k-1$ "large" integration variables are not "independent" such that $\sum_{j=1}^{N_{c}-1} x_{j}$ stays finite (this requires $k \leq N_{c}-3$ ), the number of "large" integration variables is effectively reduced by one. After a suitable change of variables we get a contribution given by (3.34) with $k$ replaced by $k+1$.

We now proceed in analogy with (3.30) and replace the coefficient functions by $\tilde{b}_{\lambda, m}(H)$ appropriate for $\mathfrak{s u}\left(N_{c}\right)$. Provided that leading-order contributions do not vanish acciden- 
tally, we end up with

$$
\gamma^{m} \int_{V\left(\gamma, \mathfrak{s u}\left(N_{c}\right)\right)} d H \operatorname{det}\left(1+H^{2}\right)^{-N_{b}} \tilde{b}_{\lambda, m}(H) \propto \gamma^{\min \left\{m,\left\{\frac{1}{2}\left(k-N_{b}\right)^{2}-\frac{1}{2}\left(N_{c}-N_{b}\right)^{2}+\frac{1}{2}\right\}_{0 \leq k \leq N_{c}-2}\right\}},
$$

unless the minimum in the exponent on the r.h.s. equals $m$ and $\frac{1}{2}\left(k-N_{b}\right)^{2}-\frac{1}{2}\left(N_{c}-N_{b}\right)^{2}+$ $\frac{1}{2}=m$ for some $k \leq N_{c}-2$, in which case the integral is proportional to $\gamma^{m} \log (\gamma)$ at leading order. Since $\frac{1}{2}\left(k-N_{b}\right)^{2}-\frac{1}{2}\left(N_{c}-N_{b}\right)^{2}+\frac{1}{2}$ is minimized by $k=\min \left\{\left[N_{b}\right], N_{c}-2\right\}$, we obtain

$$
\begin{aligned}
\gamma^{m} \int_{V\left(\gamma, \mathfrak{s u}\left(N_{c}\right)\right)} d H \operatorname{det}\left(1+H^{2}\right)^{-N_{b}} \tilde{b}_{\lambda, m}(H) \\
\propto \begin{cases}\gamma^{\frac{1}{2}\left(\left[N_{b}\right]-N_{b}\right)^{2}-\frac{1}{2}\left(N_{c}-N_{b}\right)^{2}+\frac{1}{2}} & \text { for } 0<N_{b} \leq N_{c}-\frac{3}{2} \\
\gamma^{2\left(N_{b}-\left(N_{c}-\frac{5}{4}\right)\right)} & \text { for } N_{c}-\frac{5}{2} \leq N_{b}<N_{c}-\frac{5}{4}+\frac{m}{2} \\
\gamma^{m} \log (\gamma) & \text { for } N_{b}=N_{c}-\frac{5}{4}+\frac{m}{2} \\
\gamma^{m} & \text { for } N_{c}-\frac{5}{4}+\frac{m}{2}<N_{b} .\end{cases}
\end{aligned}
$$

In complete analogy to the $\mathrm{U}\left(N_{c}\right)$ case, see the discussion below (3.31) and compare (3.36) to (3.31), we obtain

$$
\lim _{\alpha \rightarrow 1} c_{\lambda}(\alpha)=d_{\lambda} \quad \text { for } N_{b} \geq N_{c}-\frac{5}{4}
$$

while the limit will generically differ from $d_{\lambda}$ for $N_{b}<N_{c}-\frac{5}{4}$.

\subsubsection{Bounds on $N_{b}$}

In conclusion, the necessary and sufficient condition for the weight function (3.1) to reduce to a Dirac $\delta$-function on the group manifold in the $\alpha \rightarrow 1$ limit is given by

$$
N_{b} \geq \begin{cases}N_{c}-\frac{1}{2} & \text { for } G=\mathrm{U}\left(N_{c}\right) \\ N_{c}-\frac{5}{4} & \text { for } G=\mathrm{SU}\left(N_{c}\right)\end{cases}
$$

These bounds have been verified through extensive numerical simulations.

As discussed in some detail in [14], when the statistical weight in the partition function approaches a product of $\delta$-functions for the plaquette variables, correlation lengths diverge and we expect the lattice theory to converge to a continuum limit.

\subsection{Nature of the continuum limit}

In the previous section we have found that the theory defined by the weight function (3.1) admits a continuum limit if the bounds (3.38) are satisfied. To investigate the nature of this continuum limit, we now determine the next-to-leading-order (NLO) terms in the expansion of $c_{\lambda}(\alpha)$ about $\alpha=1$, i.e., NLO corrections to (3.32) and (3.37). 


\subsubsection{NLO terms for $G=\mathrm{U}\left(N_{c}\right)$}

For $G=\mathrm{U}\left(N_{c}\right)$ and $N_{b}>N_{c}-\frac{1}{2}$, the integral (3.31) is finite as $\gamma \rightarrow 0$. For $N_{b} \geq N_{c}+\frac{1}{2}$, we furthermore see that the result for $m=1$ dominates over all terms with $m>1$. This means that the NLO term in $c_{\lambda}(\alpha)$ is exclusively determined by the first-order term of the expansion of the integrand in (3.30). From the expansion (3.11) of $\chi_{\lambda}$ we thus obtain for $N_{b}>N_{c}+\frac{1}{2}$

$$
c_{\lambda}(\alpha)=\frac{\bar{c}_{\lambda}(\gamma)}{\bar{c}_{0}(\gamma)}=d_{\lambda}\left\{1-\frac{\gamma}{2}\left[\left(\frac{q(\lambda)^{2}}{N_{c}^{2}}-\frac{A(\lambda)}{N_{c}}\right)\left\langle(\operatorname{tr} H)^{2}\right\rangle_{\mathfrak{u}\left(N_{c}\right)}+A(\lambda)\left\langle\operatorname{tr} H^{2}\right\rangle_{\mathfrak{u}\left(N_{c}\right)}\right]+o(\gamma)\right\}
$$

with

$$
\langle f(H)\rangle_{\mathfrak{g}}=\frac{\int_{\mathfrak{g}} d H \operatorname{det}\left(1+H^{2}\right)^{-N_{b}} f(H)}{\int_{\mathfrak{g}} d H \operatorname{det}\left(1+H^{2}\right)^{-N_{b}}}
$$

and $o(\gamma)$ refers to the little-o notation. Note that subleading corrections to $\int_{V} d H \operatorname{det}(1+$ $\left.H^{2}\right)^{-N_{b}} \propto \gamma^{0}$ result only in terms of order $o(\gamma)$ in the ratio $\bar{c}_{\lambda} / \bar{c}_{0}$ since the integral does not depend on $\lambda$. Furthermore, contributions from $\lambda$-independent terms in $b_{\lambda, 1}(H)$ cancel at order $\gamma$ in (3.39).

For $N_{b}=N_{c}+\frac{1}{2}, \gamma$ on the r.h.s. of (3.39) has to be replaced by $\gamma \log (\gamma)$, and the $\gamma \rightarrow 0$ limit implicit in (3.40), i.e., $\lim _{\gamma \rightarrow 0} V(\gamma, \mathfrak{g})=\mathfrak{g}$, has to be taken more carefully, see (3.29) and (3.31).

For $N_{c}-\frac{1}{2} \leq N_{b}<N_{c}+\frac{1}{2}$, (3.31) yields the same leading-order term for all $m \geq$ 1, proportional to $\gamma^{N_{b}-\left(N_{c}-\frac{1}{2}\right)}$, which implies that the NLO term in $c_{\lambda}(\alpha)$ is not simply determined by the term of order $\gamma$ in the expansion (3.11) of the character $\chi_{\lambda}$.

For $\mathrm{U}(1)$, the coefficient $c_{\lambda}$ can be calculated analytically for $N_{b} \in \mathbb{N}$, confirming the results derived above (see appendix B.2). Our result (3.39) is in agreement ${ }^{5}$ with [14, eq. (25)], which was derived for integer $N_{b} \geq N_{c}+1$.

\subsubsection{NLO terms for $G=\mathrm{SU}\left(N_{c}\right)$}

In complete analogy to the $\mathrm{U}\left(N_{c}\right)$ case, see (3.31) compared to (3.36), we obtain from (3.16) for $N_{b}>N_{c}-\frac{3}{4}$

$$
c_{\lambda}(\alpha)=\frac{\bar{c}_{\lambda}(\gamma)}{\bar{c}_{0}(\gamma)}=d_{\lambda}\left[1-\gamma \frac{C_{2}^{\mathrm{SU}}(\lambda)}{N_{c}^{2}-1}\left\langle\operatorname{tr} H^{2}\right\rangle_{\mathfrak{s u}\left(N_{c}\right)}+o(\gamma)\right]
$$

For $N_{b}=N_{c}-\frac{3}{4}$, the comment made after (3.39) applies. For $N_{c}-\frac{5}{4} \leq N_{b}<N_{c}-\frac{3}{4}$, all terms in the expansion of the integrand contribute to the NLO term in (3.41), resulting in a more complicated dependence on $\lambda$ compared to (3.41). For SU(2), the coefficient $c_{\lambda}$ can be calculated analytically for $2 N_{b} \in \mathbb{N}$, confirming these results (see appendix B.1).

\footnotetext{
${ }^{5}$ From $(2.12)$ and $\alpha_{\mathrm{BZ}}=m_{\mathrm{BZ}}^{-4}$ we obtain $\gamma=\left(1-\alpha_{\mathrm{BZ}}\right)^{2} / \alpha_{\mathrm{BZ}}=\left(1-\alpha_{\mathrm{BZ}}\right)^{2}+\ldots$ Note that $X$ in $[14$, eq. (25)] corresponds to our $i H$.
} 
Assuming large $N_{b}$, we now perform a saddle-point expansion about the trivial saddle at $H=0$ and obtain

$$
\begin{aligned}
N_{b}\left\langle\operatorname{tr} H^{2}\right\rangle_{\mathfrak{s u}\left(N_{c}\right)}=\frac{1}{2}\left(N_{c}^{2}-1\right)[1 & +\left(N_{c}-\frac{3}{2 N_{c}}\right) \frac{1}{N_{b}}+\left(N_{c}^{2}-\frac{15}{4}+\frac{21}{4 N_{c}^{2}}\right) \frac{1}{N_{b}^{2}} \\
& \left.+\left(N_{c}^{3}-\frac{27 N_{c}}{4}+\frac{93}{4 N_{c}}-\frac{267}{8 N_{c}^{3}}\right) \frac{1}{N_{b}^{3}}+\mathcal{O}\left(N_{b}^{-4}\right)\right],
\end{aligned}
$$

where the effective expansion parameter appears to be $N_{c} / N_{b}$. For $\mathrm{SU}(2)$, the exact result is given by

$$
\left.\frac{\frac{1}{2}\left(N_{c}^{2}-1\right)}{N_{b}\left\langle\operatorname{tr} H^{2}\right\rangle_{\mathfrak{s u}\left(N_{c}\right)}}\right|_{N_{c}=2}=1-\frac{5}{4 N_{b}} .
$$

For small $N_{c}$, it is more convenient to use the eigenvalue parameterization of $H \in \mathfrak{s u}\left(N_{c}\right)$ (instead of the parameterization as a linear combination of generators) for the saddle-point approximation since the computation of higher-order terms can then be automated easily. In this way, we obtain

$$
\begin{aligned}
\left.\frac{\frac{1}{2}\left(N_{c}^{2}-1\right)}{N_{b}\left\langle\operatorname{tr} H^{2}\right\rangle_{\mathfrak{s u}\left(N_{c}\right)}}\right|_{N_{c}=3}= & 1-\frac{5}{2 N_{b}}+\frac{5}{12 N_{b}^{2}}+\frac{5}{18 N_{b}^{3}}-\frac{95}{432 N_{b}^{4}}-\frac{485}{2592 N_{b}^{5}}+\frac{12715}{7776 N_{b}^{6}} \\
& +\frac{127445}{93312 N_{b}^{7}}-\frac{4267895}{559872 N_{b}^{8}}+\frac{6392335}{839808 N_{b}^{9}}+\frac{1424010605}{20155392 N_{b}^{10}}+\ldots \\
\left.\frac{\frac{1}{2}\left(N_{c}^{2}-1\right)}{N_{b}\left\langle\operatorname{tr} H^{2}\right\rangle_{\mathfrak{s u}\left(N_{c}\right)}}\right|_{N_{c}=4}= & 1-\frac{29}{8 N_{b}}+\frac{9}{16 N_{b}^{2}}+\frac{81}{64 N_{b}^{3}}+\frac{207}{128 N_{b}^{4}}-\frac{27}{64 N_{b}^{5}}-\frac{14787}{4096 N_{b}^{6}}+\ldots
\end{aligned}
$$

\subsubsection{Character expansion for $\mathrm{SU}\left(N_{c}\right)$ Wilson plaquette action}

To determine the nature of the continuum limit of the boson-induced lattice gauge theory, we compare (3.41) to the corresponding expansion of the familiar $\mathrm{SU}\left(N_{c}\right)$ Wilson plaquette action, see (4.6) below. In analogy to (3.3) we expand for $U \in \mathrm{SU}\left(N_{c}\right)$

$$
\frac{1}{Z_{W}} e^{\frac{1}{g_{W}^{2}} \operatorname{tr}\left(U+U^{\dagger}-2\right)}=\sum_{\lambda} c_{\lambda}^{(W)}\left(g_{W}^{2}\right) \chi_{\lambda}(U)
$$

with normalization factor $Z_{W}$ defined in the obvious manner. Using the parameterization $U=e^{i g_{W} A}$ with $A \in \mathfrak{s u}\left(N_{c}\right)$ we obtain

$$
c_{\lambda}^{(W)}\left(g_{W}^{2}\right)=d_{\lambda}\left(1-g_{W}^{2} \frac{C_{2}^{\mathrm{SU}\left(N_{c}\right)}(\lambda)}{N_{c}^{2}-1}\left\langle\operatorname{tr} A^{2}\right\rangle_{W}+\ldots\right),
$$

where

$$
\left\langle\operatorname{tr} A^{2}\right\rangle_{W}=\frac{\int_{\mathfrak{s u}\left(N_{c}\right)} d A e^{-\operatorname{tr} A^{2}} \operatorname{tr} A^{2}}{\int_{\mathfrak{s u}\left(N_{c}\right)} d A e^{-\operatorname{tr} A^{2}}}=\frac{1}{2}\left(N_{c}^{2}-1\right)
$$


is just the leading term of $N_{b}\left\langle\operatorname{tr} H^{2}\right\rangle$ computed in section 3.3 .2 by saddle-point approximation. Truncating the expansions of $c_{\lambda}(\alpha)$ and $c_{\lambda}^{(W)}\left(g_{W}^{2}\right)$ after the NLO term, both weight factors become equivalent to the heat-kernel weight

$$
\omega_{\mathrm{HK}}(U, t)=\sum_{\lambda} d_{\lambda} e^{-t C_{2}^{\mathrm{SU}\left(N_{c}\right)}(\lambda)} \chi_{\lambda}(U)
$$

with diffusion times $t \propto \gamma \propto(1-\alpha)$ and $t \propto g_{W}^{2}$, respectively. A similar equivalence holds for $G=\mathrm{U}\left(N_{c}\right)[14]$.

\subsubsection{Continuum limit in $2 \mathrm{~d}$}

In two dimensions, the heat-kernel lattice action is exactly self-reproducing and therefore invariant under subdivision of the lattice (Migdal's recursion [22]). Consider, e.g., two neighboring plaquettes $p_{1}$ and $p_{2}$, where $p_{2}$ is to the right of $p_{1}$. If the common link variable is called $U$, the plaquette variables are parametrized as $U_{p_{1}}=V_{1} U W_{1}$ and $U_{p_{2}}=U^{\dagger} W_{2}$. Then, due to character orthogonality (3.4),

$$
\int_{G} d U \omega_{\mathrm{HK}}\left(U_{p_{1}}, t\right) \omega_{\mathrm{HK}}\left(U_{p_{2}}, t\right)=\omega_{\mathrm{HK}}\left(U_{p_{1}+p_{2}}, 2 t\right),
$$

where $U_{p_{1}+p_{2}}=V_{1} W_{2} W_{1}$ is the Wilson loop variable for the boundary of the joint lattice cell $p_{1}+p_{2}$.

If we set $t=t_{p} a^{2}$, with $t_{p}$ (of dimension 1/area) fixed in the continuum limit $a \rightarrow 0$, and consider a region $R$ of physical area $A_{R}$ in flat spacetime, obtained by gluing together $n=A_{R} / a^{2}$ elementary plaquettes, the effective action for $U_{R}$ (the product of link variables along the boundary of $R$ ), determined by integrating over all internal link variables, is given by

$$
\omega_{\mathrm{HK}}\left(U_{R}, n t\right)=\omega_{\mathrm{HK}}\left(U_{R}, A_{R} t_{p}\right)
$$

Since the effective action has the same functional form as the original plaquette action and only the diffusion parameter changes proportional to the enclosed area, taking the continuum limit is trivial in two dimensions for the heat-kernel action. In flat spacetime, Wilson loop variables (corresponding to closed non-selfintersecting curves enclosing an area $\left.A_{R}\right)$ are simply distributed according to the distribution (3.51).

From the effective action (3.51) for fundamental polygons, the YM partition function on an orientable surface of genus $g$ and dimensionless area $A$ (in suitable units) was found in $[15]$ to be given by

$$
Z_{g}(A)=\sum_{\lambda} d_{\lambda}^{2-2 g} e^{-A C_{2}^{\mathrm{SU}\left(N_{c}\right)}(\lambda)}
$$

From (3.41) and (3.47) we see that using either the induced or the Wilson weight function instead of the heat-kernel distribution for the elementary plaquette variables still leads to the effective action (3.51) in the continuum limit $a \rightarrow 0$ if we scale $\gamma \propto a^{2}$ and $g_{W}^{2} \propto a^{2}$, respectively. This implies that the continuum limit of the induced $\mathrm{SU}\left(N_{c}\right)$ theory with $N_{b} \geq$ 
$N_{c}-\frac{3}{4}$ is equivalent to YM theory in two dimensions. Similarly, we conclude from the results of section 3.3.1 that the continuum limit for $\mathrm{U}\left(N_{c}\right)$ is equivalent to $\mathrm{YM}$ for $N_{b} \geq N_{c}+\frac{1}{2}$.

To ensure that the induced action and the Wilson action lead to the same physics in the continuum limit in two dimensions we require $c_{\lambda}(\alpha)=c_{\lambda}^{(W)}\left(g_{W}^{2}\right)$ to NLO and thus obtain from (3.41) and (3.47)

$$
\begin{aligned}
\frac{1}{g_{W}^{2}}= & \frac{1}{\gamma} \frac{\left\langle\operatorname{tr} A^{2}\right\rangle_{W}}{\left\langle\operatorname{tr} H^{2}\right\rangle_{\mathfrak{s u}\left(N_{c}\right)}} \\
= & \frac{N_{b}}{\gamma}\left(1-\frac{1}{2 N_{c}}\left(2 N_{c}^{2}-3\right) \frac{1}{N_{b}}+\frac{3}{4 N_{c}^{2}}\left(N_{c}^{2}-4\right) \frac{1}{N_{b}^{2}}\right. \\
& \left.\quad+\frac{3}{4 N_{c}^{3}}\left(N_{c}^{2}-4\right)\left(N_{c}^{2}-7\right) \frac{1}{N_{b}^{3}}+\ldots\right) .
\end{aligned}
$$

This relation between the couplings is consistent with the more general result obtained from perturbation theory in an arbitrary number of dimensions, see section 4 below. In higher dimensions, taking the continuum limit is of course more involved.

\subsubsection{Bounds on $N_{b}$ in $2 \mathrm{~d}$}

In conclusion, the necessary and sufficient condition for the continuum limit of the induced theory to be equivalent to YM theory in two dimensions is

$$
N_{b} \geq \begin{cases}N_{c}+\frac{1}{2} & \text { for } G=\mathrm{U}\left(N_{c}\right), \\ N_{c}-\frac{3}{4} & \text { for } G=\mathrm{SU}\left(N_{c}\right) .\end{cases}
$$

\subsubsection{Continuum limit in $3 \mathrm{~d}$ and $4 \mathrm{~d}$}

Following [14], we conjecture that the equivalence with YM theory persists also in higher dimensions. Furthermore, the "exotic" case $N_{b}=N_{c}$ for $G=\mathrm{U}\left(N_{c}\right)$ in two dimensions, where the continuum limit of the induced theory differs from YM theory, was studied in great detail in [14]. BZ argue that this unusual theory of Cauchy-type is unlikely to persist in three or four dimensions. Similarly, we expect the continuum limit of the induced theory to be equivalent to YM theory in $d>2$ for both $G=\mathrm{U}\left(N_{c}\right)$ and $G=\mathrm{SU}\left(N_{c}\right)$ whenever the continuum limit actually exists, i.e., if (3.38) is satisfied. Our numerical tests support these expectations [16, 23].

\section{Perturbative matching of the couplings}

In the following, we consider only $G=\mathrm{SU}\left(N_{c}\right)$ since this case includes the gauge group of $\mathrm{QCD}$ and is therefore of phenomenological interest.

\subsection{General strategy}

Since the continuum limit is essentially trivial in two dimensions, the relation between $1-\alpha$ and the Wilson coupling $g_{W}^{2}$ can be obtained simply by matching the character expansions of the plaquette weight functions, resulting in (3.53) above. On the other hand, in three 
and four dimensions the continuum limit is more involved so that we need an alternative method to determine the relation between the bare couplings. A natural candidate is perturbation theory, which we will use in the following.

Ideally, one would like to expand around the continuum limit at $\alpha=1$ for fixed $N_{b}$. However, we then encounter two problems. First, the expansion of the logarithm in

$$
\begin{aligned}
S_{I} & =-\log \prod_{p} \operatorname{det}^{-N_{b}}\left(1-\frac{\alpha}{2}\left(U_{p}+U_{p}^{\dagger}\right)\right)=N_{b} \sum_{p} \operatorname{tr} \log \left(1-\frac{\alpha}{2}\left(U_{p}+U_{p}^{\dagger}\right)\right) \\
& =N_{b} \sum_{p} \operatorname{tr} \log \left(1-\frac{\alpha}{2(1-\alpha)}\left(U_{p}+U_{p}^{\dagger}-2\right)\right)
\end{aligned}
$$

where we omitted an irrelevant constant in the last step, converges only if $\left|\frac{\alpha}{1-\alpha}(\cos \varphi-1)\right| \leq$ 1 for all possible eigenvalues $e^{i \varphi}$ of $U_{p}$, i.e., if $\alpha \leq \frac{1}{3}$. Second, after expanding the logarithm anyway, we see that a saddle-point analysis of the partition function is not possible since higher orders of $U+U^{\dagger}-2$ are not suppressed in

$$
S_{I}=-N_{b} \sum_{p} \sum_{n=1}^{\infty} \frac{1}{n} \frac{1}{\gamma^{n}} \operatorname{tr}\left(U_{p}+U_{p}^{\dagger}-2\right)^{n}
$$

with $\gamma=2(1-\alpha) / \alpha$ as defined in (3.6), and we would end up with non-Gaussian integrals. ${ }^{6}$ As a workaround, we will therefore first keep $\alpha \leq \frac{1}{3}$ fixed (i.e., $\gamma \geq 4$ ) and take the limit $N_{b} \rightarrow \infty$, which allows for a systematic saddle-point analysis, and then analytically continue $g_{W}\left(\alpha, N_{b}\right)$ to small $1-\alpha$ at the end.

It is natural to define the coupling $\tilde{g}_{I}$ for the induced theory in the limit $N_{b} \rightarrow \infty$ at fixed $\alpha$ as $^{7}$

$$
\frac{1}{\tilde{g}_{I}^{2}}=N_{b} \frac{\alpha}{2(1-\alpha)}=\frac{N_{b}}{\gamma}
$$

since the induced action in terms of this coupling $\tilde{g}_{I}$ and the fixed parameter $\gamma \geq 4$ reads

$$
S_{I}=-\frac{1}{\tilde{g}_{I}^{2}}\left\{\sum_{p} \operatorname{tr}\left(U_{p}+U_{p}^{\dagger}-2\right)+\sum_{n=2}^{\infty} \sum_{p} \frac{1}{n \gamma^{n-1}} \operatorname{tr}\left(U_{p}+U_{p}^{\dagger}-2\right)^{n}\right\},
$$

where the first term is identical to the Wilson gauge action

$$
S_{W}=-\frac{1}{g_{W}^{2}} \sum_{p} \operatorname{tr}\left(U_{p}+U_{p}^{\dagger}-2\right)
$$

\footnotetext{
${ }^{6}$ For simplicity, consider only a single plaquette and parametrize $U=e^{i \sqrt{\gamma} H}$ such that the $H^{2}$-term in $S_{I}$ has a coefficient of order $\gamma^{0}$. Then, an expansion in powers of $\gamma$ results in

$$
S_{I}=-N_{b} \sum_{n=1}^{\infty} \frac{(-1)^{n}}{n} \operatorname{tr} H^{2 n}+\mathcal{O}(\gamma)=N_{b} \log \operatorname{det}\left(1+H^{2}\right)+\mathcal{O}(\gamma),
$$

as expected from (3.7). This means that all powers of $H^{2}$ contribute to the action at leading order in $\gamma$.

${ }^{7}$ One could of course choose to include subleading terms in $1 / N_{b}$ in the definition of $\tilde{g}_{I}$, but the definition in (4.4) seems to be the most natural choice.
} 
with coupling $g_{W}$ replaced by $\tilde{g}_{I}$. All sums over $p$ are sums over unoriented plaquettes, i.e., $p=(x, \mu<\nu)$. Expanding (4.5) around $U=\mathbb{1}$ in the usual manner (at fixed $\gamma$ ), we observe that the induced action $S_{I}$ reduces to the familiar YM action in the continuum limit $U \rightarrow \mathbb{1}$.

It is obvious from (4.5) that the induced action reproduces the Wilson action at the lattice level (i.e., at non-zero $g$ ) if we take the limit $\gamma \rightarrow \infty$ at fixed $\tilde{g}_{I}$, corresponding to the limit discussed in connection with (2.3). However, this is not what we are interested in here. In the following, we keep $\gamma$ fixed and expand in powers of $\tilde{g}_{I}$.

At fixed $\gamma$, the coupling $\tilde{g}_{I}$ plays a role which is in complete analogy to that of $g_{W}$ for the Wilson action. Parametrizing the link variables as $U=e^{i \tilde{g}_{I} A}$, functional integrals can be systematically expanded in powers of $\tilde{g}_{I}$ in a saddle-point analysis. A character expansion of the plaquette weight function results in coefficients $c_{\lambda}$ which are identical, up to $\mathcal{O}\left(g^{2}\right)$, to those that we obtained for the Wilson action in section 3.3.3. This implies that the weight function reduces to a $\delta$-function on the $\mathrm{SU}\left(N_{c}\right)$ manifold in the limit $\tilde{g}_{I} \rightarrow 0$ (at fixed $\gamma$ ) and that the continuum limit is equivalent to YM theory in two dimensions. Therefore, keeping $\gamma$ fixed and taking $\tilde{g}_{I} \rightarrow 0$, we expect the induced theory to describe YM theory in the continuum for all $\gamma \geq 4$ also in three and four dimensions (with a dependence on $\gamma$ occurring, e.g., in the ratio $\Lambda_{\text {lat }} / \Lambda_{\text {cont }}$ of the $\Lambda$ parameters).

Moreover, expanding the action and the partition function in $\tilde{g}_{I}$, the same powercounting arguments apply as in the familiar Wilson case. In particular, using the background-field technique, we only have to expand the action to quadratic order in the quantum fields to compute the effective two-point function for the background fields at one-loop order (which determines the ratio of the $\Lambda$ parameters).

Our aim in this part of the paper is to calculate the relation between the couplings

$$
\frac{1}{g_{W}^{2}}=\frac{1}{\tilde{g}_{I}^{2}}\left(1+c_{1}(\gamma) \tilde{g}_{I}^{2}+c_{2}(\gamma) \tilde{g}_{I}^{4}+\ldots\right)
$$

We will see in sections 4.2 .5 and 4.2 .6 below that

$$
\begin{aligned}
& c_{1}(\gamma)=\frac{c_{1,-1}}{\gamma}, \\
& c_{2}(\gamma)=\frac{c_{2,-2}}{\gamma^{2}}+\frac{c_{2,-1}}{\gamma} .
\end{aligned}
$$

The one-loop coefficient $c_{1}(\gamma)$ in (4.7) directly determines the ratio of the $\Lambda$ parameters,

$$
\frac{\Lambda_{I}(\gamma)}{\Lambda_{W}}=\exp \left(\frac{c_{1}(\gamma)}{2 \beta_{0}}\right), \quad \beta_{0}=\frac{11 N_{c}}{48 \pi^{2}}
$$

It will turn out, see (4.48) below, that $c_{1}(\gamma)$ is always negative. The two-loop coefficient $c_{2}(\gamma)$ determines the first non-universal coefficient $\beta_{2}$ in the $\beta$ function.

When we write $1 / g_{W}^{2}$ as a function of $\gamma$ and $N_{b}$, it turns out that only simple poles in $\gamma$ appear (also when we extend (4.7) to higher orders in $\tilde{g}_{I}$, see section 4.2 .4 below), i.e., $c_{n}(\gamma) \propto \gamma^{-n}$ for $\gamma \rightarrow 0$. Replacing $\tilde{g}_{I}^{2}$ by $\gamma / N_{b}$ in (4.7), we may formally regard $1 / g_{W}^{2}$ as a series in $\gamma$ with coefficients depending on $N_{b}$ (although the relation is strictly valid only 
in the limit $N_{b} \rightarrow \infty$ at fixed $\gamma \geq 4$ ). Assuming that we can analytically continue (4.7) to small $\gamma=2(1-\alpha) / \alpha$, we obtain

$$
\frac{1}{g_{W}^{2}}=\frac{d_{0}\left(N_{b}\right)}{\gamma}+d_{1}\left(N_{b}\right)+\mathcal{O}(\gamma)
$$

with

$$
\begin{aligned}
& d_{0}\left(N_{b}\right)=N_{b}+c_{1,-1}+\frac{c_{2,-2}}{N_{b}}+\mathcal{O}\left(N_{b}^{-2}\right), \\
& d_{1}\left(N_{b}\right)=\frac{c_{2,-1}}{N_{b}}+\mathcal{O}\left(N_{b}^{-2}\right) .
\end{aligned}
$$

For the limit $\gamma \rightarrow 0$ (i.e., $\alpha \rightarrow 1$ ) at fixed $N_{b}$, a natural definition of the coupling is thus given by $^{8}$

$$
\frac{1}{g_{I}^{2}}=\frac{d_{0}\left(N_{b}\right)}{\gamma}
$$

so that

$$
\frac{1}{g_{W}^{2}}=\frac{1}{g_{I}^{2}}\left(1+d_{1}\left(N_{b}\right) g_{I}^{2}+\ldots\right)
$$

In the following, we will calculate $c_{1,-1}$ and $c_{2,-2}$ using the background-field technique. The computation of the remaining two-loop coefficient $c_{2,-1}$ is considerably more involved and therefore left for future work.

\subsection{Background-field calculation}

\subsubsection{Effective action}

The background-field technique was introduced in [24]. Following [25-27], we define the effective action

$$
e^{-\Gamma[A]} \propto \int_{1 \mathrm{PI}}[D q] e^{-S[A, q]},
$$

where $A$ is the background field, $q$ is the quantum field, and the path integral is over one-particle irreducible graphs with an arbitrary number of external lines. Here, $A$ is not required to satisfy the YM field equations. We will expand only to quadratic order in $A$ since this is sufficient to determine the relation of the couplings. In the expansion of the action $S$, terms linear in $q$ can be omitted since they do not contribute to 1PI diagrams.

The gauge-fixing procedure for the induced theory can be taken over one-to-one from the Wilson case. It is convenient to use the background-field gauge since the effective action $\Gamma[A]$ is then invariant under formal background-field transformations (resulting in constraints on renormalization parameters). We argue below that diagrams with ghost loops cannot contribute to $c_{1,-1}$ and $c_{2,-2}$. Integrals over ghost fields are therefore already omitted in (4.16). Also, we can ignore the renormalization of the gauge-fixing parameter

\footnotetext{
${ }^{8}$ Again, it is possible to include subleading terms in $\gamma$ in the definition of $g_{I}$, effectively changing the coefficients $d_{j}(j \geq 1)$.
} 
since (i) we will not compute $c_{2,-1}$ and (ii) the coefficient $c_{2,-2}$ is determined exclusively by the two-point function of the background field, see section 4.2.4.

The relation between the couplings $g_{W}$ and $\tilde{g}_{I}$ is obtained by requiring $\Gamma_{I}[A]=\Gamma_{W}[A]$ in the continuum limit $g \rightarrow 0$. To compute the effective action, we expand $S[A, q]$ (including gauge-fixing terms) in powers of the quantum field $q$, separate the classical piece (i.e., terms independent of $q$ ) and the free part (i.e., terms of order $A^{0} q^{2}$ ) of the action from interaction terms (i.e., all other terms) and compute their (one-particle irreducible connected) expectation values w.r.t. the free action,

$$
\Gamma[A]=S_{\mathrm{cl}}[A]-\sum_{k=1}^{\infty} \frac{1}{k !}\left\langle\left(-S_{\mathrm{int}}[A, q]\right)^{k}\right\rangle_{1 \mathrm{PI}-\mathrm{C}} .
$$

Since we are only interested in the two-point function for the background field it is sufficient to calculate expectation values of $S_{\text {int }}^{k}$ at order $A^{2}$. Requiring $\Gamma_{I}[A]=\Gamma_{W}[A]$ in the continuum limit will result in an equation of the form

$$
\frac{1}{g_{W}^{2}}+c_{1}^{(W)}+c_{2}^{(W)} g_{W}^{2}+\mathcal{O}\left(g_{W}^{4}\right)=\frac{1}{\tilde{g}_{I}^{2}}+\left(c_{1}^{(W)}+c_{1}(\gamma)\right)+\left(c_{2}^{(W)}+c_{2}(\gamma)\right) \tilde{g}_{I}^{2}+\mathcal{O}\left(\tilde{g}_{I}^{4}\right),
$$

where we have split the coefficients for the induced theory into contributions that originate exclusively from the Wilson part of the action and terms that depend on $\gamma$. Since $g_{W}^{2}=$ $\tilde{g}_{I}^{2}+\mathcal{O}\left(\tilde{g}_{I}^{4}\right)$ we end up with (4.7). Obviously, $c_{1}^{(W)}$ drops out of (4.18), and $c_{2}^{(W)}$ drops out in $\mathcal{O}\left(g^{2}\right)$ and therefore does not need to be computed explicitly.

\subsubsection{Expansion of the gauge action}

We parametrize the link variables as $[26,27]^{9}$

$$
U_{\mu}(x)=U_{\mu}^{(0)}(x) e^{i a g q_{\mu}(x)}, \quad U_{\mu}^{(0)}(x)=e^{i a A_{\mu}(x)},
$$

where we imply $g=\tilde{g}_{I}$ or $g=g_{W}$ for the induced action and the Wilson action, respectively. Since we need to expand the gauge action only to quadratic order in $A$, we write

$$
S_{I}=\left.S_{W}\right|_{g_{W}=\tilde{g}_{I}}+\sum_{n=2}^{\infty}\left(S_{I}^{(n, 0)}+S_{I}^{(n, 1)}+S_{I}^{(n, 2)}+\mathcal{O}\left(A^{3}\right)\right)
$$

where $S_{I}^{(n, k)}$ includes all $\mathcal{O}\left(A^{k}\right)$ terms resulting from $\operatorname{tr}\left(U_{p}+U_{p}^{\dagger}-2\right)^{n}$ in the sum over $n$ in (4.5). Defining

$$
\begin{gathered}
q_{\mu \nu}(x)=q_{\mu}(x)+q_{\nu}(x+\mu)-q_{\mu}(x+\nu)-q_{\nu}(x), \\
A_{\mu \nu}(x)=A_{\mu}(x)+A_{\nu}(x+\mu)-A_{\mu}(x+\nu)-A_{\nu}(x)
\end{gathered}
$$

\footnotetext{
${ }^{9}$ Note that (4.19) corresponds to [27, eq. (4)] with $q_{\mu}=-\alpha_{\mu}$ and $A_{\mu}=-W_{\mu}$ since $U_{\mu}(x)=U(x, x+\mu)^{\dagger}$.
} 
we obtain to leading order in the quantum field (see appendix $\mathrm{C}$ for details)

$$
\begin{aligned}
S_{I}^{(n, 0)}= & (-1)^{n+1} \frac{a^{2 n} g^{2 n-2}}{\gamma^{n-1}} \sum_{x, \mu, \nu} \frac{1}{2 n} \operatorname{tr}\left[q_{\mu \nu}(x)^{2 n}+\mathcal{O}\left(g q^{2 n+1}\right)\right], \\
S_{I}^{(n, 1)}= & (-1)^{n+1} \frac{a^{2 n} g^{2 n-3}}{\gamma^{n-1}} \sum_{x, \mu, \nu} \operatorname{tr}\left[A_{\mu \nu}(x) q_{\mu \nu}(x)^{2 n-1}+\mathcal{O}\left(g A q^{2 n}\right)\right], \\
S_{I}^{(n, 2)}= & (-1)^{n+1} \frac{a^{2 n} g^{2 n-4}}{\gamma^{n-1}} \sum_{x, \mu, \nu} \operatorname{tr}\left[\frac{1}{2}\left(A_{\mu \nu}(x) q_{\mu \nu}(x)^{n-1}\right)^{2}\right. \\
& \left.+\sum_{m=0}^{n-2} A_{\mu \nu}(x) q_{\mu \nu}(x)^{m} A_{\mu \nu}(x) q_{\mu \nu}(x)^{2 n-m-2}+\mathcal{O}\left(g A^{2} q^{2 n-1}\right)\right] .
\end{aligned}
$$

\subsubsection{Gauge fixing and free action for the quantum field}

For the expansion of $S_{W}[U]$ in terms of $A$ and $q$ using the parameterization (4.19), as well as for the gauge-fixing procedure, we can use the results of $[26,27]$. Since we do not have to compute $c_{1,2}^{(W)}$ in (4.18) to determine the relation between $g_{W}$ and $\tilde{g}_{I}$, all we need here is the free (gauge-fixed) action for the quantum field $q$ to quadratic order.

The gauge-fixing term in background-field Feynman gauge is given by

$$
S_{\mathrm{gf}}=a^{4} \sum_{x} \operatorname{tr}\left(\sum_{\mu} \bar{D}_{\mu}^{(0)} q_{\mu}\right)^{2}
$$

with the lattice covariant derivative (involving only the background field)

$$
\bar{D}_{\mu}^{(0)} q_{\nu}(x)=\frac{1}{a}\left(U_{\mu}^{(0)}(x-\mu) q_{\nu}(x-\mu) U_{\mu}^{(0) \dagger}(x-\mu)-q_{\nu}(x)\right) .
$$

The free action for the quantum field $q$ is obtained by combining $\left.S_{\mathrm{gf}}\right|_{A=0}$ with the terms of order $A^{0} q^{2}$ in the gauge action $S_{I}$. The latter are obtained from $S_{I}^{(1,0)}$, which is defined in the sentence following (4.20) and given explicitly in (4.23). $S_{I}^{(1,0)}$ is part of $S_{W}$ on the r.h.s. of (4.20). This means that the free action is identical for the induced and the Wilson gauge action. It is found to be given by

$$
S_{f}=a^{4} \sum_{x, \mu, \nu} \operatorname{tr}\left(\Delta_{\mu} q_{\nu}(x)\right)^{2}=a^{4} \sum_{x, \nu} \operatorname{tr}\left(q_{\nu}(x) \square q_{\nu}(x)\right)
$$

with lattice derivatives

$$
\begin{aligned}
\Delta_{\mu} f(x) & =a^{-1}(f(x+\mu)-f(x)), \\
\bar{\Delta}_{\mu} f(x) & =a^{-1}(f(x-\mu)-f(x)), \\
\square & =\sum_{\mu} \bar{\Delta}_{\mu} \Delta_{\mu} .
\end{aligned}
$$

Writing $q_{\mu}$ as a linear combination of $\mathrm{SU}\left(N_{c}\right)$ generators, $q_{\mu}=\sum_{b=1}^{N_{c}^{2}-1} q_{\mu}^{b} t_{b}$, we obtain with the normalization condition (A.1)

$$
S_{f}=\frac{a^{4}}{2} \sum_{x, \mu, b} q_{\mu}^{b}(x) \square q_{\mu}^{b}(x),
$$


which is just the free action of a collection of $d\left(N_{c}^{2}-1\right)$ independent massless scalar fields. Here, $d$ denotes the number of Euclidean spacetime dimensions. The free propagator is therefore given by

$$
D_{\mu \nu}^{a b}(x, y)=\left\langle q_{\mu}^{a}(x) q_{\nu}^{b}(y)\right\rangle=\delta_{a b} \delta_{\mu \nu} D(x-y)
$$

with the standard lattice propagator for a massless scalar field

$$
D(x-y)=\int_{-\pi / a}^{\pi / a} \frac{d^{d} p}{(2 \pi)^{d}} e^{i p(x-y)} \frac{a^{d-2}}{\sum_{\mu} 2\left(1-\cos \left(a p_{\mu}\right)\right)} .
$$

For our calculation, it will be convenient to define

$$
\left\langle q_{\mu_{1} \nu_{1}}^{a_{1}}\left(x_{1}\right) q_{\mu_{2} \nu_{2}}^{a_{2}}\left(x_{2}\right)\right\rangle=\delta_{a_{1} a_{2}} D_{\mu_{1} \nu_{1}, \mu_{2} \nu_{2}}\left(x_{1}-x_{2}\right)
$$

for $q_{\mu \nu}$ given in (4.21). Using

$$
q_{\mu \nu}(x)=a\left(\Delta_{\mu} q_{\nu}(x)-\Delta_{\nu} q_{\mu}(x)\right)
$$

we obtain

$$
D_{\mu_{1} \nu_{1}, \mu_{2} \nu_{2}}(z)=a^{2}\left(\delta_{\nu_{1} \nu_{2}} \Delta_{\mu_{1}} \bar{\Delta}_{\mu_{2}}+\delta_{\mu_{1} \mu_{2}} \Delta_{\nu_{1}} \bar{\Delta}_{\nu_{2}}-\delta_{\nu_{1} \mu_{2}} \Delta_{\mu_{1}} \bar{\Delta}_{\nu_{2}}-\delta_{\mu_{1} \nu_{2}} \Delta_{\nu_{1}} \bar{\Delta}_{\mu_{2}}\right) D(z) .
$$

Using the background-field gauge ensures that $\Gamma$ is a gauge-invariant functional of $A$. Assuming the background fields to be small and slowly varying as usual, this implies that in the continuum limit the lowest-order term is proportional to $\operatorname{tr} F_{\mu \nu}^{2}$. In the following, we will only focus on terms of order $A^{2}$ in $\Gamma$ (i.e., we do not explicitly check that the linear term vanishes) and identify $\left(\partial_{\mu} A_{\nu}-\partial_{\nu} A_{\mu}\right)^{2}$ with $F_{\mu \nu}^{2}$.

Note that the expectation value of a product of an odd number of $q$ fields vanishes. Therefore odd powers of $g$ are absent in (4.18).

\subsubsection{Power counting}

Counting powers of $\tilde{g}_{I}$ and $\gamma$ in expectation values (w.r.t. the free action for the quantum field ${ }^{10}$ ) of products of the form

$$
S_{I}^{(l, 2)} \prod_{i}\left(S_{I}^{\left(n_{i}, 0\right)}\right)^{m_{i}}, \quad S_{I}^{\left(l_{1}, 1\right)} S_{I}^{\left(l_{2}, 1\right)} \prod_{i}\left(S_{I}^{\left(n_{i}, 0\right)}\right)^{m_{i}}
$$

we see that there are terms of order $A^{2}$ in $\Gamma[A]$ with coefficients of order

$$
\frac{1}{\tilde{g}_{I}^{2}}\left(\frac{\tilde{g}_{I}^{2}}{\gamma}\right)^{n}\left(1+\mathcal{O}\left(\tilde{g}_{I}^{2}\right)\right)=\frac{1}{\gamma} \frac{1}{N_{b}^{n-1}}\left(1+\mathcal{O}\left(\frac{\gamma}{N_{b}}\right)\right), \quad n \geq 0
$$

with $n=l-1+\sum_{i} m_{i}\left(n_{i}-1\right)$ and $n=l_{1}+l_{2}-2+\sum_{i} m_{i}\left(n_{i}-1\right)$, respectively. We observe that only simple poles in $\gamma$ will appear when we write the coefficients in terms of $\gamma$

\footnotetext{
${ }^{10}$ The free action is obtained from the term of order $A^{0} q^{2}$ in $S_{I}^{(1,0)}$, which is of order $g^{0} \gamma^{0}$. Therefore, free propagators do not lead to additional factors of $g$ or $\gamma$, see (4.33) and (4.34).
} 
and $N_{b}$, see section 4.1. Furthermore, the residue at the pole is exclusively determined by expectation values of the form (4.38), where only the leading terms of $S_{I}^{(n, k)}$ given in (4.23) through (4.25) contribute. Subleading terms result in corrections of order $\gamma^{m}$ with $m \geq 0$ on the r.h.s. of eq. (4.39).

Since the Wilson gauge action is given by the (implicitly defined) $n=1$ term in (4.5), one might think that the leading terms of $S_{I}^{(1, k)}$ with $k=0,1,2$ would contribute to the residue at the pole in the effective action. However, the leading term of $S_{I}^{(1,0)}$ is quadratic in the quantum field $q$ and therefore only contributes to the free action. For $S_{I}^{(1,1)}$, the leading term is linear in $q$ and therefore does not contribute to one-particle irreducible diagrams (the same applies to the first subleading term of $S_{I}^{(1,2)}$ ). Finally, the leading term of $S_{I}^{(1,2)}$ is just the classical piece of the effective action.

It is obvious that diagrams containing measure vertices or ghost loops cannot contribute to the coefficient of the pole in $\gamma$ in the two-point function of the background field since these vertices appear with powers of $\tilde{g}_{I}^{2}=\gamma / N_{b}$ without any accompanying factors of $1 / \gamma$.

Expectation values that involve only terms from $S_{I}^{(n=1)}$ and $S_{\text {gf }}$ do not depend on $\gamma$ and are collected in $c_{m}^{(W)}$ at order $\left(\tilde{g}_{I}^{2}\right)^{m-1}$ on the r.h.s. of (4.18).

In summary, when the r.h.s. of (4.18) is written in terms of $N_{b}$ and $\gamma$, the coefficient of the pole in $\gamma$ is determined exclusively by expectation values of products of leading terms of $S_{I}^{(n, k)}$ with $n \geq 2$. This means that we do not have to consider mixing of the Wilson part of the induced action or $S_{\text {gf }}$ with $n \geq 2$ terms in expectation values of $S_{\text {int }}^{k}$, see (4.5), (4.17), and (4.38).

In order to determine the full two-loop coefficient $c_{2}(\gamma)$ in the relation of $g_{W}$ and $\tilde{g}_{I}$ in (4.7) one also has to take into account the renormalization of the gauge parameter, which is obtained from the gluon self energy at one-loop order [28]. However, we note that this does not result in a contribution to the pole coefficient $c_{2,-2}$. The reason is again a factor of $\tilde{g}_{I}^{2}$ without any accompanying factor of $1 / \gamma$. The pole coefficients $c_{1,-1}$ and $c_{2,-2}$ can therefore be determined exclusively from the two-point function of the background field, by requiring $\Gamma_{I}[A]=\Gamma_{W}[A]$ at order $A^{2}$ with $\Gamma[A]$ obtained through (4.17).

\subsubsection{Effective action to one loop}

To determine the coefficient of order $\tilde{g}_{I}^{0}$ in (4.18), we have to take into account terms of

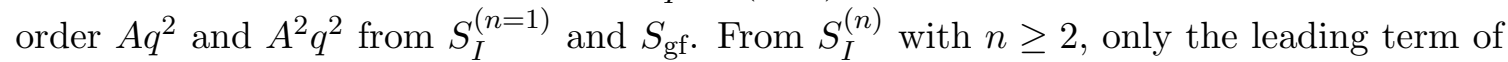
$S_{I}^{(2,2)}$ contributes, which is of order $A^{2} q^{2}$. Therefore, terms from $S_{I}^{(n=1)}$ and $S_{\mathrm{gf}}$ determine $c_{1}(W)$ but do not contribute to $c_{1}(\gamma)$ on the r.h.s. of (4.18). Hence, for the one-loop coefficient $c_{1}(\gamma)$, we only have to calculate the expectation value of

$$
S_{I}^{(2,2)}=-\frac{a^{4}}{\gamma} \sum_{x} \sum_{\mu, \nu} \operatorname{tr}\left[\left(A_{\mu \nu}(x)\right)^{2}\left(q_{\mu \nu}(x)\right)^{2}+\frac{1}{2} A_{\mu \nu}(x) q_{\mu \nu}(x) A_{\mu \nu}(x) q_{\mu \nu}(x)\right]
$$


see (4.25). Expanding in terms of $\mathrm{SU}\left(N_{c}\right)$ generators, we have ${ }^{11}$

$$
\begin{aligned}
\left\langle S_{I}^{(2,2)}\right\rangle & =-\frac{a^{4}}{\gamma} \sum_{x} \sum_{\mu, \nu} A_{\mu \nu}^{a}(x) A_{\mu \nu}^{b}(x)\left\langle q_{\mu \nu}^{c}(x) q_{\mu \nu}^{d}(x)\right\rangle \operatorname{tr}\left[t_{a} t_{b} t_{c} t_{d}+\frac{1}{2} t_{a} t_{c} t_{b} t_{d}\right] \\
& =-\frac{a^{4}}{\gamma} \sum_{x} \sum_{\mu, \nu} A_{\mu \nu}^{a}(x) A_{\mu \nu}^{b}(x) D_{\mu \nu, \mu \nu}(0) \operatorname{tr}\left[t_{a} t_{b} t_{c} t_{c}+\frac{1}{2} t_{a} t_{c} t_{b} t_{c}\right]
\end{aligned}
$$

since the propagator for $q_{\mu}$ is diagonal in color space, see (4.35). Making use of the identities provided in appendix A immediately results in

$$
\begin{aligned}
& s_{a b}^{(2,2)}=\operatorname{tr}\left[t_{a} t_{b} t_{c} t_{c}+\frac{1}{2} t_{a} t_{c} t_{b} t_{c}\right]=\left(\frac{N_{c}^{2}-1}{2 N_{c}}-\frac{1}{4 N_{c}}\right) \operatorname{tr}\left(t_{a} t_{b}\right)=s^{(2,2)} \delta_{a b}, \\
& s^{(2,2)}=\frac{2 N_{c}^{2}-3}{8 N_{c}} .
\end{aligned}
$$

Due to $A_{\mu \mu}=0$ we can restrict the sum over $\mu$ and $\nu$ to $\mu \neq \nu$. For this case, we obtain from (4.37)

$$
D_{\mu \nu, \mu \nu}(0)=a^{2}\left(\Delta_{\mu} \bar{\Delta}_{\mu}+\Delta_{\nu} \bar{\Delta}_{\nu}\right) D(0) .
$$

Since $\Delta_{\mu} \bar{\Delta}_{\mu} D(0)$ is independent of $\mu$ due to hypercubic symmetry, we get

$$
D_{\mu \nu, \mu \nu}(0)=a^{2} \frac{2}{d} \square D(0)=\frac{2}{d a^{2}} .
$$

In the continuum limit, we can identify $a^{-1} A_{\mu \nu}(x)$ with the field strength tensor $-F_{\mu \nu}(x)$ (since the effective action has to be gauge invariant) and end up with

$$
\left\langle S_{I}^{(2,2)}\right\rangle=-\frac{4}{d} s^{(2,2)} \frac{1}{\gamma} a^{4-d} \int d^{d} x \sum_{\mu, \nu} \operatorname{tr} F_{\mu \nu}(x)^{2}+\ldots
$$

Taking the continuum limit of $S_{I}^{(1,2)}$ in $(4.25)$ we obtain

$$
S_{\mathrm{cl}}[A]=\frac{1}{2 \tilde{g}_{I}^{2}} a^{4-d} \int d^{d} x \sum_{\mu, \nu} \operatorname{tr} F_{\mu \nu}(x)^{2}+\ldots
$$

which results in, see (4.18),

$$
c_{1}(\gamma)=-\frac{8}{d} s^{(2,2)} \frac{1}{\gamma}=-\frac{2 N_{c}^{2}-3}{N_{c} d} \frac{1}{\gamma} .
$$

Using (4.8) this means

$$
c_{1,-1}=-\frac{2 N_{c}^{2}-3}{N_{c} d} .
$$

\footnotetext{
${ }^{11}$ Sums over repeated color indices are always implied.
} 


\subsubsection{Relevant two-loop contributions}

From the general discussion in section 4.2.4 we know that, in order to obtain the coefficient $c_{2,-2}$ in (4.9), we only have to compute the contribution to $\left\langle S_{\text {int }}-\frac{1}{2} S_{\text {int }}^{2}\right\rangle$ given by the leading term of

$$
\left\langle S_{I}^{(3,2)}-S_{I}^{(2,0)} S_{I}^{(2,2)}-\frac{1}{2} S_{I}^{(2,1)} S_{I}^{(2,1)}\right\rangle
$$

(a) Expectation value of the (3, 2)-term. From (4.25) we obtain

$$
\begin{aligned}
S_{I}^{(3,2)}=\frac{a^{6} \tilde{g}_{I}^{2}}{\gamma^{2}} \sum_{x, \mu, \nu} \operatorname{tr}[ & A_{\mu \nu}(x)^{2} q_{\mu \nu}(x)^{4}+A_{\mu \nu}(x) q_{\mu \nu}(x) A_{\mu \nu}(x) q_{\mu \nu}(x)^{3} \\
& \left.+\frac{1}{2} A_{\mu \nu}(x) q_{\mu \nu}(x)^{2} A_{\mu \nu}(x) q_{\mu \nu}(x)^{2}\right] .
\end{aligned}
$$

Since

$$
\left\langle q_{\mu \nu}^{c}(x) q_{\mu \nu}^{d}(x) q_{\mu \nu}^{e}(x) q_{\mu \nu}^{f}(x)\right\rangle=\left(\delta_{c d} \delta_{e f}+\delta_{c e} \delta_{d f}+\delta_{c f} \delta_{d e}\right) D_{\mu \nu, \mu \nu}(0)^{2},
$$

see (4.35), we need to calculate the trace

$$
s_{a b}^{(3,2)}=\left(\delta_{c d} \delta_{e f}+\delta_{c e} \delta_{d f}+\delta_{c f} \delta_{d e}\right) \operatorname{tr}\left[t_{a} t_{b} t_{c} t_{d} t_{e} t_{f}+t_{a} t_{c} t_{b} t_{d} t_{e} t_{f}+\frac{1}{2} t_{a} t_{c} t_{d} t_{b} t_{e} t_{f}\right]
$$

with sums over repeated color indices implied as usual. Using (A.3) and (A.4) we obtain

$$
s_{a b}^{(3,2)}=s^{(3,2)} \delta_{a b}, \quad s^{(3,2)}=\frac{5}{16}\left(N_{c}^{2}-3+\frac{3}{N_{c}^{2}}\right) .
$$

With (4.45) we then obtain

$$
\left\langle S_{I}^{(3,2)}\right\rangle=\frac{\tilde{g}_{I}^{2}}{\gamma^{2}} \frac{8 s^{(3,2)}}{d^{2}} a^{2} \sum_{x, \mu, \nu} \operatorname{tr} A_{\mu \nu}(x)^{2},
$$

and replacing $A_{\mu \nu} \rightarrow-a F_{\mu \nu}$ in the continuum limit results in

$$
\left\langle S_{I}^{(3,2)}\right\rangle=\frac{\tilde{g}_{I}^{2}}{\gamma^{2}} \frac{8 s^{(3,2)}}{d^{2}} a^{4-d} \int d^{d} x \sum_{\mu \nu} \operatorname{tr} F_{\mu \nu}(x)^{2}+\ldots
$$

(b) Expectation value of the (2, 2; 2, 0)-term. For $\left\langle S_{I}^{(2,2)} S_{I}^{(2,0)}\right\rangle$ we have to compute the 1 PI connected expectation value

$$
\left\langle q_{\mu \nu}^{a}(x) q_{\mu \nu}^{b}(x) q_{\mu \nu}^{c}(x) q_{\mu \nu}^{d}(x) q_{\rho \lambda}^{e}(y) q_{\rho \lambda}^{f}(y)\right\rangle_{1 \mathrm{PI}-\mathrm{C}}=D_{\mu \nu, \rho \lambda}(x-y)^{2} D_{\mu \nu, \mu \nu}(0) f_{a b c d e f}
$$

with

$$
\begin{aligned}
f_{a b c d e f}= & \delta_{e a}\left(\delta_{f b} \delta_{c d}+\delta_{f c} \delta_{b d}+\delta_{f d} \delta_{b c}\right)+\delta_{e b}\left(\delta_{f a} \delta_{c d}+\delta_{f c} \delta_{a d}+\delta_{f d} \delta_{a c}\right) \\
& +\delta_{e c}\left(\delta_{f a} \delta_{b d}+\delta_{f b} \delta_{a d}+\delta_{f d} \delta_{a b}\right)+\delta_{e d}\left(\delta_{f a} \delta_{b c}+\delta_{f b} \delta_{a c}+\delta_{f c} \delta_{a b}\right)
\end{aligned}
$$


We define the color factor as

$$
s_{i j}^{(2,2 ; 2,0)}=f_{a b c d e f} \operatorname{tr}\left[t_{a} t_{b} t_{c} t_{d}\right] \operatorname{tr}\left[t_{i} t_{j} t_{e} t_{f}+\frac{1}{2} t_{i} t_{e} t_{j} t_{f}\right]
$$

and obtain, by repeatedly using (A.3) and (A.4),

$$
s_{i j}^{(2,2 ; 2,0)}=s^{(2,2 ; 2,0)} \delta_{i j}, \quad s^{(2,2 ; 2,0)}=2\left(\frac{2 N_{c}^{2}-3}{4 N_{c}}\right)^{2} .
$$

After some algebra we obtain

$$
\sum_{\mu, \nu} \sum_{\rho, \lambda} \operatorname{tr} A_{\rho \lambda}(y)^{2} \sum_{x} D_{\mu \nu, \rho \lambda}(x)^{2} D_{\mu \nu, \mu \nu}(0)=\frac{8}{a^{6} d^{2}} \sum_{\rho, \lambda} \operatorname{tr} A_{\rho \lambda}(y)^{2},
$$

which leads to

$$
\left\langle S_{I}^{(2,0)} S_{I}^{(2,2)}\right\rangle_{1 \mathrm{PI}-\mathrm{C}}=\frac{\tilde{g}_{I}^{2}}{\gamma^{2}} \frac{4 s^{(2,2 ; 2,0)}}{d^{2}} a^{2} \sum_{x, \mu, \nu} \operatorname{tr} A_{\mu \nu}(x)^{2} .
$$

In the continuum limit this yields

$$
\left\langle S_{I}^{(2,0)} S_{I}^{(2,2)}\right\rangle_{1 \mathrm{PI}-\mathrm{C}}=\frac{\tilde{g}_{I}^{2}}{\gamma^{2}} \frac{4 s^{(2,2 ; 2,0)}}{d^{2}} a^{4-d} \int d^{d} x \sum_{\mu, \nu} \operatorname{tr} F_{\mu \nu}(x)^{2}+\ldots
$$

(c) Expectation value of the $(2,1 ; 2,1)$-term. For the contribution of $\left\langle S_{I}^{(2,1)} S_{I}^{(2,1)}\right\rangle$ we only need to compute the 1PI connected part

$$
\left\langle q_{\mu \nu}^{a}(x) q_{\mu \nu}^{b}(x) q_{\mu \nu}^{c}(x) q_{\rho \lambda}^{d}(y) q_{\rho \lambda}^{e}(y) q_{\rho \lambda}^{f}(y)\right\rangle_{1 \mathrm{PI}-\mathrm{C}}=D_{\mu \nu, \rho \lambda}(x-y)^{3} g_{a b c d e f}
$$

with

$$
g_{a b c d e f}=\delta_{a d} \delta_{b e} \delta_{c f}+\delta_{a d} \delta_{b f} \delta_{c e}+\delta_{a e} \delta_{b d} \delta_{c f}+\delta_{a e} \delta_{b f} \delta_{c d}+\delta_{a f} \delta_{b d} \delta_{c e}+\delta_{a f} \delta_{b e} \delta_{c d} .
$$

Thus, we need to evaluate

$$
s_{i j}^{(2,1 ; 2,1)}=g_{a b c d e f} \operatorname{tr}\left[t_{i} t_{a} t_{b} t_{c}\right] \operatorname{tr}\left[t_{j} t_{d} t_{e} t_{f}\right] .
$$

Using (A.3) and (A.4) we obtain after some algebra

$$
s_{i j}^{(2,1 ; 2,1)}=s^{(2,1 ; 2,1)} \delta_{i j}, \quad s^{(2,1 ; 2,1)}=\frac{N_{c}^{4}-6 N_{c}^{2}+18}{16 N_{c}^{2}} .
$$

We assume the background field to be slowly varying [26] and expand $A_{\rho \lambda}(y) A_{\mu \nu}(x)$ around a common point, e.g., $x$, effectively substituting $A_{\rho \lambda}(y) \rightarrow A_{\rho \lambda}(x)$ at leading order. Then we only need to compute

$$
\sum_{\mu \neq \nu, \rho \neq \lambda} \operatorname{tr}\left[A_{\mu \nu}(x) A_{\rho \lambda}(x)\right] \sum_{y} D_{\mu \nu, \rho \lambda}(y)^{3} .
$$


Since the effective background-field action is gauge invariant, all contributions with $\{\mu, \nu\} \neq$ $\{\rho, \lambda\}$ vanish. After some algebra we obtain

$$
\sum_{\mu \neq \nu, \rho \neq \lambda} \operatorname{tr}\left[A_{\mu \nu}(x) A_{\rho \lambda}(x)\right] \sum_{y} D_{\mu \nu, \rho \lambda}(y)^{3}=a^{-6} C_{d} \sum_{\mu \neq \nu} \operatorname{tr} A_{\mu \nu}(x)^{2}
$$

with

$$
\begin{aligned}
C_{d} & =\frac{4}{d-1}\left(\frac{3}{d^{2}}-4(4-d) J_{d}\right), \\
J_{d} & =\frac{1}{8} \int_{-\pi}^{\pi} \frac{d^{d} k}{(2 \pi)^{d}} \frac{d^{d} q}{(2 \pi)^{d}} \frac{\left(\sin \left(k_{1}\right)+\sin \left(q_{1}\right)-\sin \left(k_{1}+q_{1}\right)\right)^{2}}{\sum_{\gamma, \mu, \rho}\left(1-\cos \left(k_{\gamma}\right)\right)\left(1-\cos \left(q_{\mu}\right)\right)\left(1-\cos \left(k_{\rho}+q_{\rho}\right)\right)} .
\end{aligned}
$$

For $d=2$, we find after some algebra $J_{d=2}=\frac{1}{32}$. For $d=3$ we have evaluated the integral $J_{d}$ numerically and obtained $J_{d=3} \approx 0.0085535415$. This results in

$$
C_{d=4}=\frac{1}{4}, \quad C_{d=3} \approx 0.59823833, \quad C_{d=2}=2
$$

and leads to

$$
\begin{aligned}
\frac{1}{2}\left\langle S_{I}^{(n=2, k=1)} S_{I}^{(n=2, k=1)}\right\rangle_{1 \mathrm{PI}} & =\frac{\tilde{g}_{I}^{2}}{\gamma^{2}} s^{(2,1 ; 2,1)} C_{d} a^{2} \sum_{x, \mu, \nu} \operatorname{tr} A_{\mu \nu}(x)^{2} \\
& =\frac{\tilde{g}_{I}^{2}}{\gamma^{2}} s^{(2,1 ; 2,1)} C_{d} a^{4-d} \int d^{d} x \sum_{\mu, \nu} \operatorname{tr} F_{\mu \nu}(x)^{2}+\ldots
\end{aligned}
$$

(d) Coefficient $\boldsymbol{c}_{\mathbf{2},-\mathbf{2}}$. From (4.9), (4.18), (4.47), (4.56), (4.63), and (4.73) we finally obtain

$$
\begin{aligned}
c_{2,-2} & =\frac{8}{d^{2}}\left(2 s^{(3,2)}-s^{(2,2 ; 2,0)}\right)-2 s^{(2,1 ; 2,1)} C_{d} \\
& =\frac{N_{c}^{4}-3 N_{c}^{2}+6}{d^{2} N_{c}^{2}}-\frac{N_{c}^{4}-6 N_{c}^{2}+18}{2 N_{c}^{2}(d-1)}\left(\frac{3}{d^{2}}-4(4-d) J_{d}\right)
\end{aligned}
$$

and in particular

$$
\begin{aligned}
& \left.c_{2,-2}\right|_{d=4}=\frac{N_{c}^{4}-6}{32 N_{c}^{2}}, \\
& \left.c_{2,-2}\right|_{d=3}=\frac{N_{c}^{4}+6 N_{c}^{2}-30}{36 N_{c}^{2}}+\frac{N_{c}^{4}-6 N_{c}^{2}+18}{N_{c}^{2}} J_{3}, \\
& \left.c_{2,-2}\right|_{d=2}=\frac{3}{4}-\frac{3}{N_{c}^{2}}
\end{aligned}
$$

with $J_{d}$ from (4.71) and $J_{3} \approx 0.0085535415$.

For $d=2$, the results for $c_{1,-1}$ and $c_{2,-2}$ are consistent with (3.53), which was obtained by matching the character expansions of the plaquette weight functions for the Wilson action and the induced action in the continuum limit. 


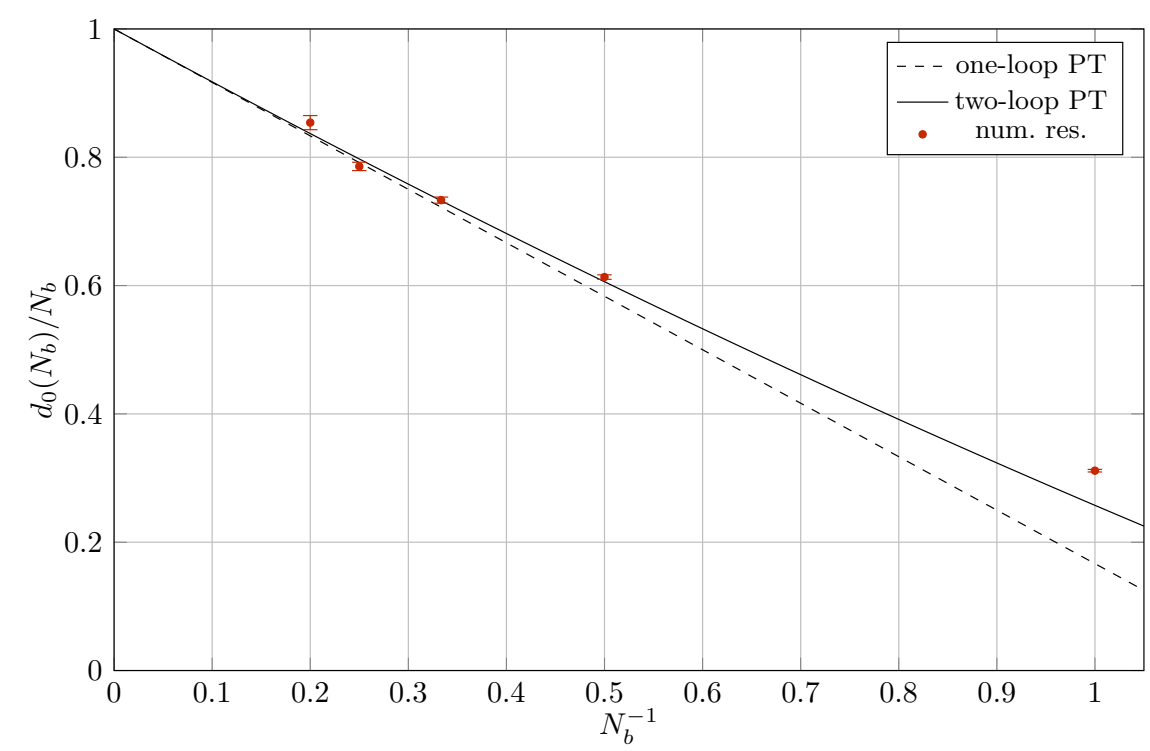

Figure 2. Perturbative and numerical results for $d_{0} / N_{b}$ in $d=3$ with $N_{c}=2$.

\subsection{Comparison with numerical results}

Using the methods and results introduced in [16], we determined the coefficient $d_{0}$ in (4.14) numerically through simulations with both Wilson and induced gauge action for $N_{c}=2$ in three dimensions. ${ }^{12}$ Using (4.49) and (4.76) for $d=3$ and $N_{c}=2$, the perturbative expansion of $d_{0}$ in (4.12) reads

$$
\frac{d_{0}\left(N_{b}\right)}{N_{b}}=1-\frac{5}{6 N_{b}}+\frac{0.0908283}{N_{b}^{2}}+\mathcal{O}\left(N_{b}^{-3}\right) .
$$

The numerical results are shown in figure 2 together with the perturbative results. Note that the latter were derived assuming large $N_{b}$ and $\gamma \geq 4$. Nevertheless we observe very good agreement even for small $N_{b}$ and small $\gamma$, i.e., outside the domain of validity of (4.78).

Note also that the numerical results include the value $N_{b}=1$, which is outside the bound (3.54) (for the continuum limit to be equivalent to YM theory in $d=2$ ) but inside the bound (3.38) (for the continuum limit to exist at all). The fact that the corresponding data point is close to the perturbative results is consistent with our expectation (formulated in section 3.3.6) that the continuum limit of the induced theory is equivalent to YM theory in $d>2$ whenever the continuum limit actually exists.

\section{Conclusions}

In this paper we have explored the novel approach of Budzcies and Zirnbauer [14] to induced QCD, which represents a major step forward compared to earlier approaches since it requires only a small number $N_{b}$ of auxiliary bosons. We slightly reformulated the action

\footnotetext{
${ }^{12}$ This was done by first matching the bare couplings of both approaches through the determination of the Sommer parameter $r_{0}$ [29]. Then the data were fitted to (4.11), including the $\mathcal{O}(\gamma)$ term. We have simulated at couplings corresponding to $0.116 \leq \gamma \leq 3.26$. The details of the simulations and the numerical results will be discussed in [23].
} 
to solve a trivial sign problem present in the original formulation. We then extended the analysis of [14] from gauge group $\mathrm{U}\left(N_{c}\right)$ to $\mathrm{SU}\left(N_{c}\right)$. The latter case is of particular interest since it includes the gauge group of QCD. We derived refined bounds on $N_{b}$, admitting also non-integer values, for the induced theory to have a continuum limit at fixed $N_{b}$ and for this continuum limit to be in the universality class of YM theory in two dimensions. We conjectured that in higher dimensions the latter bounds can be relaxed. We also performed a perturbative calculation using the background-field technique to match the bare coupling of the induced theory to the standard lattice coupling. Formally, the result of this calculation is only valid in the continuum limit $N_{b} \rightarrow \infty$ at fixed $\alpha \leq \frac{1}{3}$. The latter condition excludes the "interesting" continuum limit $\alpha \rightarrow 1$ at fixed $N_{b}$. However, by comparing to data from lattice-gauge simulations near the continuum limit, we observe very good agreement also for parameters outside the formal range of validity. This leads us to conjecture that the perturbative results are also valid in the "interesting" continuum limit $\alpha \rightarrow 1$.

In future work, we will present detailed numerical evidence from lattice simulations in three and four dimensions that standard lattice gauge theory and induced theory (at fixed $N_{b}$ ) have the same continuum limit, and that away from the continuum limit they differ only by relatively small lattice artifacts. The numerical simulations include quantities at both zero and non-zero temperature.

Having presented analytical and numerical evidence in support of the induced theory, an important question is to what extent this new approach is useful in the sense that it leads to better simulation algorithms or new formulations that would allow us to go beyond what is possible in the standard formulation. To this end we will explore a dual formulation of lattice gauge theory, including fermions, in which the gauge field is integrated out first. This can be done since it only appears linearly in the action. After integrating out the fermions, the remaining path integral over the auxiliary boson fields involves only gauge-invariant objects. It will be interesting to see whether a worm-like algorithm can be constructed for this dual formulation.

\section{A Color traces}

The traceless generators $t_{a}$ in the fundamental representation of $\mathrm{SU}\left(N_{c}\right)$ are normalized according to

$$
\operatorname{tr}\left(t_{a} t_{b}\right)=\frac{1}{2} \delta_{a b}
$$

and obey

$$
\sum_{c}\left(t_{c}\right)_{i j}\left(t_{c}\right)_{k l}=\frac{1}{2}\left(\delta_{i l} \delta_{j k}-\frac{1}{N_{c}} \delta_{i j} \delta_{k l}\right), \quad \sum_{c} t_{c} t_{c}=\frac{N_{c}^{2}-1}{2 N_{c}} .
$$

For arbitrary matrices $A$ and $B$ we therefore have

$$
\begin{aligned}
\sum_{c} \operatorname{tr}\left(A t_{c} B t_{c}\right) & =\frac{1}{2}(\operatorname{tr} A)(\operatorname{tr} B)-\frac{1}{2 N_{c}} \operatorname{tr}(A B), \\
\sum_{c} \operatorname{tr}\left(A t_{c}\right) \operatorname{tr}\left(B t_{c}\right) & =-\frac{1}{2 N_{c}}(\operatorname{tr} A)(\operatorname{tr} B)+\frac{1}{2} \operatorname{tr}(A B) .
\end{aligned}
$$




\section{B Character expansion for $\mathrm{SU}(2)$ and $\mathrm{U}(1)$}

\section{B.1 SU(2)}

Every element $U$ of $\mathrm{SU}(2)$ can be diagonalized according to $U=V \operatorname{diag}\left(e^{i \varphi}, e^{-i \varphi}\right) V^{\dagger}$ with $V$ unitary and $\varphi \in[-\pi, \pi]$. In the character expansion we only integrate over class functions, which are independent of $V$. It therefore suffices to integrate over $U=e^{i \varphi \sigma_{3}}$ with measure

$$
d \mu(\varphi)=\frac{1}{\pi} \sin ^{2} \varphi d \varphi
$$

The dimension $d_{k}$, the quadratic $\mathrm{SU}(2)$ Casimir operator $C_{2}(k)$, and the character $\chi_{k}$ for the irreducible representation corresponding to a one-row Young diagram with $k$ boxes are given by

$$
\begin{aligned}
d_{k} & =k+1, \\
C_{2}(k) & =\frac{1}{4} k(k+2), \\
\chi_{k}(\varphi) & =\frac{\sin ((k+1) \varphi)}{\sin \varphi} .
\end{aligned}
$$

Since

$$
\operatorname{det}\left(1-\frac{\alpha}{2}\left(U+U^{\dagger}\right)\right)=(1-\alpha \cos \varphi)^{2} \propto\left(\left(1-b e^{i \varphi}\right)\left(1-b e^{-i \varphi}\right)\right)^{2}
$$

with

$$
\alpha=\frac{2 b}{1+b^{2}}, \quad b=\frac{1}{\alpha}\left(1-\sqrt{1-\alpha^{2}}\right),
$$

we write the unnormalized weight function as

$$
\bar{\omega}(\varphi)=\frac{1}{\left(\left(1-b e^{i \varphi}\right)\left(1-b e^{-i \varphi}\right)\right)^{2 N_{b}}}
$$

for convenience. Expanding in characters, we have

$$
\bar{\omega}(\varphi)=\sum_{n=0}^{\infty} \bar{c}_{n} \chi_{n}(\varphi), \quad \bar{c}_{n}=\int_{-\pi}^{\pi} d \mu(\varphi) \bar{\omega}(\varphi) \chi_{n}(\varphi) .
$$

For the properly normalized weight function

$$
\omega(\varphi)=\frac{\bar{\omega}(\varphi)}{\int d \mu(\varphi) \bar{\omega}(\varphi)}=\sum_{n=0}^{\infty} c_{n} \chi_{n}(\varphi)
$$

we obtain the expansion coefficients $c_{n}=\bar{c}_{n} / \bar{c}_{0}$. Using the series expansion

$$
\frac{1}{\left(1-b e^{i \varphi}\right)^{2 N_{b}}}=\sum_{k=0}^{\infty}\left(\begin{array}{c}
2 N_{b}+k-1 \\
k
\end{array}\right) b^{k} e^{i k \varphi}, \quad\left(\begin{array}{c}
n \\
m
\end{array}\right)=\frac{\Gamma(n+1)}{\Gamma(m+1) \Gamma(n-m+1)},
$$


which is valid for $b<1$, i.e., $\alpha<1$, and in which $2 N_{b}$ is not necessarily restricted to integer values, we obtain after some algebra

$$
\bar{c}_{n}=(n+1) b^{n} \sum_{m=0}^{\infty} b^{2 m} \frac{1}{m+n+1}\left(\begin{array}{c}
2 N_{b}-2+m \\
m
\end{array}\right)\left(\begin{array}{c}
2 N_{b}-1+m+n \\
m+n
\end{array}\right) .
$$

If $2 N_{b} \in \mathbb{N}$, the infinite sum can be calculated analytically. In the following we consider the first few cases explicitly.

(i) For $N_{b}=\frac{1}{2}$ we obtain

$$
\bar{c}_{n}=b^{n}, \quad c_{n}=b^{n} .
$$

In the limit $\alpha \rightarrow 1$ (i.e., $b \rightarrow 1$ ) we have $c_{n}=1 \neq d_{n}$, and therefore $\omega$ does not reduce to a $\delta$-function on the $\mathrm{SU}(2)$ manifold, as expected for $N_{b}<\frac{3}{4}$.

(ii) For $N_{b}=1$ we obtain

$$
\begin{aligned}
& \bar{c}_{n}=(n+1) \frac{b^{n}}{1-b^{2}}, \\
& c_{n}=(n+1) b^{n}=d_{n}(1-n(1-b)+\ldots)=d_{n}(1-n \sqrt{2(1-\alpha)}+\ldots) .
\end{aligned}
$$

Taking $\alpha \rightarrow 1$, the leading term is given by $d_{n}$, but the coefficient of the next-order term is not proportional to $C_{2}$, as expected for $\frac{3}{4} \leq N_{b}<\frac{5}{4}$.

(iii) For $N_{b}=\frac{3}{2}$ we obtain

$$
\begin{aligned}
& \bar{c}_{n}=(n+1) \frac{b^{n}}{\left(1-b^{2}\right)^{3}}\left(1+\frac{n}{2}\left(1-b^{2}\right)\right), \\
& c_{n}=d_{n}\left(1-2 C_{2}(n)(1-b)^{2}+\ldots\right)=d_{n}\left(1-4 C_{2}(n)(1-\alpha)+\ldots\right)
\end{aligned}
$$

in agreement with (3.41) and (3.43).

(iv) For $N_{b}=2$ we obtain

$$
\begin{aligned}
& \bar{c}_{n}=\frac{(n+1) b^{n}}{6\left(1-b^{2}\right)^{5}}\left((n+3)(n+2)+2(n+3)(1-n) b^{2}-n(1-n) b^{4}\right), \\
& c_{n}=d_{n}\left(1-\frac{2}{3} C_{2}(n)(1-b)^{2}+\ldots\right)=d_{n}\left(1-\frac{4}{3} C_{2}(n)(1-\alpha)+\ldots\right)
\end{aligned}
$$

in agreement with (3.41) and (3.43).

For non-integer $2 N_{b}, c_{n}$ can be easily expanded around $\alpha=1$ numerically. Our numerical results are consistent with

$$
\frac{c_{n}}{d_{n}}= \begin{cases}1-\frac{4}{4 N_{b}-5} C_{2}(n)(1-\alpha)+\mathcal{O}\left((1-\alpha)^{\min \left(\frac{3}{2}, 2 N_{b}-\frac{3}{2}\right)}\right) & \text { for } N_{b}>\frac{5}{4}, \\ 1+2 C_{2}(n)(1-\alpha) \log (1-\alpha)+\ldots & \text { for } N_{b}=\frac{5}{4}, \\ 1-f(n)(1-\alpha)^{2 N_{b}-\frac{3}{2}}+\ldots & \text { for } \frac{3}{4}<N_{b}<\frac{5}{4}, \\ b^{n} \frac{\Gamma\left(\frac{3}{2}+n\right)_{2} F_{1}\left(\frac{1}{2}, \frac{3}{2}+n ; 2+n ; b^{2}\right)}{\Gamma(n+2) \Gamma\left(\frac{3}{2}\right)_{2} F_{1}\left(\frac{1}{2}, \frac{3}{2} ; 2 ; b^{2}\right)}=1-\frac{g(n)}{\log (1-\alpha)}+\ldots & \text { for } N_{b}=\frac{3}{4},\end{cases}
$$

where $f(n)$ and $g(n)$ are not proportional to $C_{2}(n)$. 


\section{B.2 U(1)}

The irreducible representations of $\mathrm{U}(1)$ are one-dimensional and characterized by $\chi_{n}(\varphi)=$ $e^{i n \varphi}$ with $n \in \mathbb{Z}$. Proceeding analogously to the $\mathrm{SU}(2)$ case discussed in detail in the previous subsection, we obtain

$$
\bar{c}_{n}=b^{|n|} \sum_{m=0}^{\infty} b^{2 m}\left(\begin{array}{c}
N_{b}+m-1 \\
m
\end{array}\right)\left(\begin{array}{c}
N_{b}+m+|n|-1 \\
m+|n|
\end{array}\right) .
$$

For $N_{b} \in \mathbb{N}$, the infinite sum can be calculated analytically, and we obtain for the first few cases

$$
c_{n}=\frac{\bar{c}_{n}}{\bar{c}_{0}}= \begin{cases}b^{|n|}=1-|n| \sqrt{\gamma}+\ldots & \text { for } N_{b}=1, \\ 1-\frac{\gamma}{2} n^{2}+\ldots & \text { for } N_{b}=2, \\ 1-\frac{\gamma}{6} n^{2}+\ldots & \text { for } N_{b}=3, \\ 1-\frac{\gamma}{10} n^{2}+\ldots & \text { for } N_{b}=4 .\end{cases}
$$

\section{Explicit expansion of the induced gauge action}

For an unoriented plaquette $p=(x, \mu<\nu)$, we write

$$
U_{p}=U_{\mu \nu}(x)=U_{\nu}^{\dagger}(x) U_{\mu}^{\dagger}(x+\nu) U_{\nu}(x+\mu) U_{\mu}(x) .
$$

Using (4.19), we then expand in powers of the background field (up to quadratic order),

$$
U_{\mu \nu}(x)+U_{\mu \nu}(x)^{\dagger}-2=C_{\mu \nu}^{(0)}(x)+C_{\mu \nu}^{(1)}(x)+C_{\mu \nu}^{(2)}(x)+\mathcal{O}\left(A^{3}\right),
$$

where the $C_{\mu \nu}^{(i)}(x)$ are of order $A^{i}$. Due to the invariance of the trace under cyclic permutations, we then get for $n \geq 2$ (omitting obvious indices and arguments)

$$
\begin{aligned}
\operatorname{tr}\left(U_{p}+U_{p}^{\dagger}-2\right)^{n}=\operatorname{tr}[ & \left(C^{(0)}\right)^{n}+n C^{(1)}\left(C^{(0)}\right)^{n-1}+n C^{(2)}\left(C^{(0)}\right)^{n-1} \\
& \left.+\frac{n}{2} \sum_{m=0}^{n-2} C^{(1)}\left(C^{(0)}\right)^{m} C^{(1)}\left(C^{(0)}\right)^{n-m-2}\right]+\mathcal{O}\left(A^{3}\right) .
\end{aligned}
$$

The last term can also be rewritten as

$$
\begin{aligned}
\frac{n}{2} \operatorname{tr} \sum_{m=0}^{n-2} C^{(1)}\left(C^{(0)}\right)^{m} C^{(1)}\left(C^{(0)}\right)^{n-m-2}= & -\frac{n}{2} \delta_{0, n \bmod 2} \operatorname{tr} C^{(1)}\left(C^{(0)}\right)^{\frac{n}{2}-1} C^{(1)}\left(C^{(0)}\right)^{\frac{n}{2}-1} \\
& +n \operatorname{tr} \sum_{m=0}^{\left\lfloor\frac{n}{2}\right\rfloor-1} C^{(1)}\left(C^{(0)}\right)^{m} C^{(1)}\left(C^{(0)}\right)^{n-m-2}
\end{aligned}
$$


Next, we expand the $C^{(i)}$ to leading order in $g q$. For arbitrary non-commuting matrices $M_{i}(i=1, \ldots, k)$ we have

$$
\begin{aligned}
e^{M_{1}} e^{M_{2}} \cdots e^{M_{k}}+e^{-M_{k}} \cdots e^{-M_{2}} e^{-M_{1}}-2 & =\sum_{i=1}^{k} M_{i}^{2}+\sum_{i<j} M_{i} M_{j}+\sum_{i>j} M_{i} M_{j}+\mathcal{O}\left(M^{3}\right) \\
& =\left(\sum_{i=1}^{k} M_{i}\right)^{2}+\mathcal{O}\left(M^{3}\right)
\end{aligned}
$$

If we apply (C.5) to the l.h.s. of (C.2) we obtain

$$
U_{\mu \nu}(x)+U_{\mu \nu}(x)^{\dagger}-2=\left[(i a g) q_{\mu \nu}(x)+(i a) A_{\mu \nu}(x)\right]^{2}+\mathcal{O}\left(A^{3}, g q A^{2},(g q)^{2} A,(g q)^{3}\right)
$$

with $q_{\mu \nu}(x)$ and $A_{\mu \nu}(x)$ defined in (4.21) and (4.22), respectively. The leading-order terms in $C^{(i)}$ can be read off from (C.6) as

$$
\begin{aligned}
& C_{\mu \nu}^{(0)}(x)=-a^{2} g^{2} q_{\mu \nu}(x)^{2}+\mathcal{O}\left((g q)^{3}\right), \\
& C_{\mu \nu}^{(1)}(x)=-a^{2} g\left(q_{\mu \nu}(x) A_{\mu \nu}(x)+A_{\mu \nu}(x) q_{\mu \nu}(x)\right)+\mathcal{O}\left((g q)^{2}\right), \\
& C_{\mu \nu}^{(2)}(x)=-a^{2} A_{\mu \nu}(x)^{2}+\mathcal{O}(g q) .
\end{aligned}
$$

Analogously, the leading terms in the expansion (C.3) can be read off from the expansion of $\operatorname{tr}\left(i a g q_{\mu \nu}+i a A_{\mu \nu}\right)^{2 n}$ up to $\mathcal{O}\left(A^{2}\right)$. Explicitly, we have

$$
\begin{aligned}
\operatorname{tr}\left(C_{\mu \nu}^{(0)}(x)\right)^{n} & =(-1)^{n} a^{2 n} g^{2 n} \operatorname{tr} q_{\mu \nu}(x)^{2 n}+\mathcal{O}\left((g q)^{2 n+1}\right), \\
n \operatorname{tr}\left[C_{\mu \nu}^{(1)}(x)\left(C_{\mu \nu}^{(0)}(x)\right)^{n-1}\right] & =(-1)^{n} a^{2 n} g^{2 n-1} 2 n \operatorname{tr}\left[A_{\mu \nu}(x) q_{\mu \nu}(x)^{2 n-1}\right]+\mathcal{O}\left((g q)^{2 n}\right),
\end{aligned}
$$

and, combining terms of order $A^{2}$,

$$
\begin{aligned}
& \operatorname{tr}\left[n C_{\mu \nu}^{(2)}(x)\left(C_{\mu \nu}^{(0)}(x)\right)^{n-1}+\frac{n}{2} \sum_{m=0}^{n-2} C_{\mu \nu}^{(1)}(x)\left(C_{\mu \nu}^{(0)}(x)\right)^{m} C_{\mu \nu}^{(1)}(x)\left(C_{\mu \nu}^{(0)}(x)\right)^{n-m-2}\right] \\
& =(-1)^{n} a^{2 n} g^{2 n-2} n \sum_{m=0}^{2 n-2} \operatorname{tr}\left[A_{\mu \nu}(x) q_{\mu \nu}(x)^{m} A_{\mu \nu}(x) q_{\mu \nu}(x)^{2 n-m-2}\right]+\mathcal{O}\left((g q)^{2 n-1}\right) \\
& =(-1)^{n} a^{2 n} g^{2 n-2} 2 n \operatorname{tr}\left[\sum_{m=0}^{n-1} A_{\mu \nu}(x) q_{\mu \nu}(x)^{m} A_{\mu \nu}(x) q_{\mu \nu}(x)^{2 n-m-2}-\frac{1}{2}\left(A_{\mu \nu}(x) q_{\mu \nu}(x)^{n-1}\right)^{2}\right] \\
& +\mathcal{O}\left((g q)^{2 n-1}\right),
\end{aligned}
$$

where we have made use of (C.4) to obtain the last expression. Taking into account the prefactors in (4.5), we end up with $S_{I}^{(n, k=0,1,2)}$ given in (4.23) through (4.25).

\section{Acknowledgments}

This work was supported by DFG in the framework of SFB/TRR-55. We thank Christoph Lehner for useful discussions. We also thank Philippe de Forcrand and Hélvio Vairinhos for discussions on their unpublished work. 
Open Access. This article is distributed under the terms of the Creative Commons Attribution License (CC-BY 4.0), which permits any use, distribution and reproduction in any medium, provided the original author(s) and source are credited.

\section{References}

[1] J.M. Blairon, R. Brout, F. Englert and J. Greensite, Chiral symmetry breaking in the action formulation of lattice gauge theory, Nucl. Phys. B 180 (1981) 439 [INSPIRE].

[2] H. Kluberg-Stern, A. Morel, O. Napoly and B. Petersson, Spontaneous chiral symmetry breaking for a $\mathrm{U}(N)$ gauge theory on a lattice, Nucl. Phys. B 190 (1981) 504 [InSPIRE].

[3] N. Kawamoto and J. Smit, Effective lagrangian and dynamical symmetry breaking in strongly coupled lattice QCD, Nucl. Phys. B 192 (1981) 100 [INSPIRE].

[4] P. Rossi and U. Wolff, Lattice QCD with fermions at strong coupling: a dimer system, Nucl. Phys. B 248 (1984) 105 [INSPIRE].

[5] F. Karsch and K.H. Mutter, Strong coupling QCD at finite baryon number density, Nucl. Phys. B 313 (1989) 541 [INSPIRE].

[6] M. Bander, Equivalence of lattice gauge and spin theories, Phys. Lett. B 126 (1983) 463.

[7] H.W. Hamber, Lattice gauge theories at Large- $N_{f}$, Phys. Lett. B 126 (1983) 471 [InSPIRE].

[8] V.A. Kazakov and A.A. Migdal, Induced QCD at large-N, Nucl. Phys. B 397 (1993) 214 [hep-th/9206015] [INSPIRE].

[9] A. Hasenfratz and P. Hasenfratz, The equivalence of the $\mathrm{SU}(N)$ Yang-Mills theory with a purely fermionic model, Phys. Lett. B 297 (1992) 166 [hep-lat/9207017] [INSPIRE].

[10] I.I. Kogan, G.W. Semenoff and N. Weiss, Induced $Q C D$ and hidden local $Z_{N}$ symmetry, Phys. Rev. Lett. 69 (1992) 3435 [hep-th/9206095] [InSPIRE].

[11] S. Elitzur, Impossibility of spontaneously breaking local symmetries, Phys. Rev. D 12 (1975) 3978 [INSPIRE].

[12] D.J. Gross, Some remarks about induced QCD, Phys. Lett. B 293 (1992) 181 [hep-th/9208002] [INSPIRE].

[13] N. Weiss, Status of the Kazakov-Migdal model, hep-th/9501060 [INSPIRE].

[14] J. Budczies and M.R. Zirnbauer, Howe duality for an induced model of lattice $\mathrm{U}(N)$ Yang-Mills theory, math-ph/0305058 [INSPIRE].

[15] E. Witten, On quantum gauge theories in two-dimensions, Commun. Math. Phys. 141 (1991) 153.

[16] B.B. Brandt and T. Wettig, Induced QCD with two auxiliary bosonic fields, PoS (LATTICE2014) 307 [arXiv: 1411.3350] [INSPIRE].

[17] B.B. Brandt, R. Lohmayer and T. Wettig, Induced YM theory with auxiliary bosons, PoS (LATTICE2015) 276 [arXiv: 1511.08374] [INSPIRE].

[18] K.G. Wilson, Confinement of quarks, Phys. Rev. D 10 (1974) 2445 [InSPIRE].

[19] H. Rothe, Lattice gauge theories: an introduction, World Scientific, Singapore (2005).

[20] H. Weyl, The classical groups, Princeton University Press, Princeton U.S.A. (1997). 
[21] A.M. Perelomov and V.M. Popov, Casimir operators for the unitary group, JETP Lett. 1 (1965) 160.

[22] A.A. Migdal, Recursion equations in gauge theories, Sov. Phys. JETP 42 (1975) 413 [INSPIRE].

[23] B.B. Brandt, R. Lohmayer and T. Wettig, Induced QCD II: numerical results, to be published.

[24] B.S. DeWitt, Quantum theory of gravity. 2. The manifestly covariant theory, Phys. Rev. 162 (1967) 1195 [INSPIRE].

[25] S. Weinberg, The quantum theory of fields. Volume 2: modern applications, Cambridge University Press, Cambridge U.K. (2005).

[26] R.F. Dashen and D.J. Gross, The relationship between lattice and continuum definitions of the gauge theory coupling, Phys. Rev. D 23 (1981) 2340 [INSPIRE].

[27] A. Hasenfratz and P. Hasenfratz, The scales of euclidean and hamiltonian lattice QCD, Nucl. Phys. B 193 (1981) 210 [INSPIRE].

[28] M. Lüscher and P. Weisz, Computation of the relation between the bare lattice coupling and the MS coupling in $\mathrm{SU}(N)$ gauge theories to two loops, Nucl. Phys. B 452 (1995) 234 [hep-lat/9505011] [INSPIRE].

[29] R. Sommer, A New way to set the energy scale in lattice gauge theories and its applications to the static force and $\alpha_{s}$ in $\mathrm{SU}(2)$ Yang-Mills theory, Nucl. Phys. B 411 (1994) 839 [hep-lat/9310022] [INSPIRE]. 